\title{
An Iterative Phase-Space Explicit Discontinuous Galerkin Method for Stellar Radiative Transfer in Extended Atmospheres*
}

\author{
Valmor F. de Almeida \\ OAK Ridge National Laboratory \\ OAK RIDGE, TN 37831-6181, USA
}

Technical Report ORNL/TM-2003/072

20 May 2003

*Also available by request to dealmeidav@ornl.gov or at URL: dealmeidav.ornl.gov. 


\begin{abstract}
A phase-space discontinuous Galerkin (PSDG) method is presented for the solution of stellar radiative transfer problems. It allows for greater adaptivity than competing methods without sacrificing generality. The method is extensively tested on a spherically symmetric, static, inverse-power-law scattering atmosphere. Results for different sizes of atmospheres and intensities of scattering agreed with asymptotic values. The exponentially decaying behavior of the radiative field in the diffusive-transparent transition region and the forward peaking behavior at the surface of extended atmospheres were accurately captured. The integrodifferential equation of radiation transfer is solved iteratively by alternating between the radiative pressure equation and the original equation with the integral term treated as an energy density source term. In each iteration, the equations are solved via an explicit, flux-conserving, discontinuous Galerkin method. Finite elements are ordered in wave fronts perpendicularly to the characteristic curves so that elemental linear algebraic systems are solved quickly by sweeping the phase space element by element. Two implementations of a diffusive boundary condition at the origin are demonstrated wherein the finite discontinuity in the radiative intensity is accurately captured by the proposed method. This allows for a consistent mechanism to preserve photon luminosity. The method was proved to be robust and fast, and a case is made for the adequacy of parallel processing. In addition to classical two-dimensional plots, results of normalized radiative intensity were mapped onto a log-polar surface exhibiting all distinguishing features of the problem studied.
\end{abstract}




\section{Contents}

List of figures

List of tables

\begin{tabular}{lll}
\hline & Introduction & 1
\end{tabular}

\begin{tabular}{|lll}
2 & The spherical-geometry transfer problem & 4
\end{tabular}

2.1 Asymptotic analytical results . . . . . . . . . . . . . . . 6

2.1.1 Radiative equilibrium . . . . . . . . . . . . . . . . 6

2.1 .2 Luminosity . . . . . . . . . . . . . . . . . . . . 6

2.1 .3 Radiative Stress . . . . . . . . . . . . . . . . . . . . . . . . . . . . . . . . . .

2.1 .4 Diffusive limit . . . . . . . . . . . . . . . . . . . . 8

2.1 .5 Streaming limit . . . . . . . . . . . . . . . . . . . 8

2.2 The inner boundary condition . . . . . . . . . . . . . . . . . 10

2.3 Scaling and working formulae . . . . . . . . . . . . . . . . . . . 11

2.3 .1 Transfer problem . . . . . . . . . . . . . . . . . . . . . 11

2.3 .2 Luminosity and stress . . . . . . . . . . . . . . . . . . . . 12

$2.3 .3 \quad$ Streaming limit . . . . . . . . . . . . . . . . . . . . . . . . . . . . . . . . . . . . . . .

2.3 .4 Diffusive limit . . . . . . . . . . . . . . . . . . . . . . . . . . . 12

2.3 .5 Radial optical depth . . . . . . . . . . . . . . . . . 13

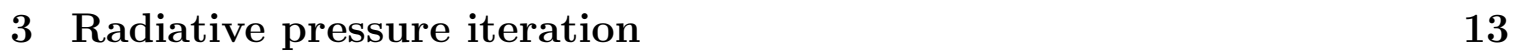

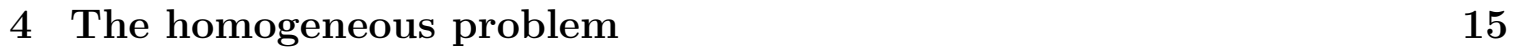

4.1 A discontinuous Galerkin finite element method . . . . . . . . . . . . 18

4.2 Computational wave fronts and elemental linear systems . . . . . . . 21

4.3 Finite element computation of moments . . . . . . . . . . . . . . 23

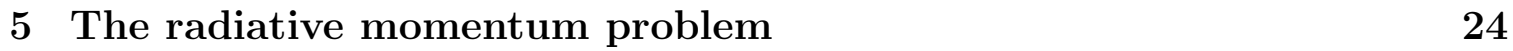

6 Results and discussion $\quad 25$

6.1 Phase space finite element partition . . . . . . . . . . . . . . . 26

6.2 Specific radiative intensity . . . . . . . . . . . . . . . . . . . 26

6.3 Energy and derived quantities . . . . . . . . . . . . . . . . . . . . 35

6.4 Convergence . . . . . . . . . . . . . . . . . . . 38

\begin{tabular}{lll}
\hline 7 & Conclusions & 38
\end{tabular}

\begin{tabular}{|ll}
\hline Acknowledgments & 41
\end{tabular}

\begin{tabular}{ll}
\hline References & 42
\end{tabular} 


\section{List of Figures}

2.1 Domain of the atmosphere . . . . . . . . . . . . . . . . . 5

4.1 Phase space characteristic lines . . . . . . . . . . . . . . . . . . 17

4.2 Computational wave fronts . . . . . . . . . . . . . . . . . . . . . . . . . . . . . . . . . 22

4.3 Finite element computation of moments . . . . . . . . . . . . . . . . 24

5.1 Inherited pressure mesh . . . . . . . . . . . . . . . . . . . 25

6.1 Stellar atmosphere sizes analyzed . . . . . . . . . . . . . . . . . . . 28

6.2 Radial meshes for all cases studied . . . . . . . . . . . . . . . . . . . 29

6.3 Angular meshes for all cases studied . . . . . . . . . . . . . . . . . . . 30

6.4 Log-polar transformation of the phase space . . . . . . . . . . . . . . 31

6.5 Normalized specific radiative intensity . . . . . . . . . . . . . . . . 32

6.6 Normalized specific intensity for various power law exponents. . . . . . 33

6.7 Limb darkening and law of darkening . . . . . . . . . . . . . . . . . 34

6.8 Undiluted energy density and pressure . . . . . . . . . . . . . . . . . . . 35

6.9 Undiluted energy density and flux (luminosity) . . . . . . . . . . . . 36

6.10 Undiluted invariants of the radiative stress tensor $\ldots . . . .37$

6.11 Eddington factor . . . . . . . . . . . . . . . . . . . . . . . 38

6.12 Updates and Galerkin (weighted) residuals convergence for $n=2$. . 39

6.13 Order of convergence . . . . . . . . . . . . . . . . . 40

\section{List of Tables}

6.1 Optical depths of inner boundaries and radius of transition region . . 26

6.2 CPU time and memory usage . . . . . . . . . . . . . . 27 


\section{Introduction}

Computational methods for radiative transfer in extended stellar atmospheres (at$U$ mospheres whose curvature of the outer layers is not negligible) require a higher degree of accuracy (compared to engineering applications on earth) to resolve the specific radiative intensity in the angular direction(s) because of the astronomical length scale, the peculiar properties of the participating media, and the complexity of the transfer processes. A chief example is the modeling of a core collapse supernova explosion (MEZZACAPPA, 2001; WOOSLEY AND HEGER, 2002). In connection with this phenomenon, a problem of radiative transfer in extended stellar atmospheres (CHANDRASEKHAR, 1945) is revisited with a phase-space discontinuous Galerkin (PSDG) method that captures salient features of the solution common to complex models of stellar astrophysics. As a bonus, the proposed method provides a general framework that can fit naturally into existing Galerkin solution methods for advanced models of stellar flows.

The radiative specific intensity in extended atmospheres possesses two distinguishing features (sec. 2), namely, exponential decay near the diffusive-transparent transition region, and forward peaking (delta-like function shape) at the surface of the atmosphere. A singularity also exists at the origin where the radiative intensity, the energy density, and the pressure grow unbounded. These features emerge in the solution of contrived forms of the general radiative transfer equation (Boltzmann equation) allowing the evaluation of improved solution methods without the difficulties of solving an elaborated realistic model.

Early studies of radiative transfer in a semi-infinite, plane-parallel stellar atmospheres led to the development of numerous approximate solution methods by many investigators. The most significant emerged from the solution of a mathematically similar neutron transport problem by Gauss quadrature (WICK, 1943). The method enjoyed the physical significance of approximating the radiation intensity by a finite set of beams aligned in the Gauss point directions - an interpretation already familiar in the early kinetic theory of gases where rudimentary models of a gas allowed a molecule to move only in few directions (see comments on Joule's attempts by TruesDel, , 1975). ChandRASEKHAR (1944) presented the method in the astrophysical context and extended it to spherical atmospheres (CHANDRASEKHAR, 1945) where the angular derivative of the radiative intensity was optimally determined as a function of the intensity value at Gauss points. In the following years, extensive investigations devoted to plane-parallel atmospheres appeared (CHANDRASEKHAR, 1948).

WICK CHANDRASEKHAR s original method was analytical wherein successive approximations with increasing accuracy were obtained by increasing the number of Gauss points; typically, the fourth approximation delivered satisfactory convergence 
for the cases studied. Computer-aided developments (often in conjunction with alternative quadrature integration rules) improved the method to become what is known as the discrete ordinates method in disciplines of radiative transfer, e.g., astrophysics and engineering heat transfer (SIEGEL AND HOWELL, 1992), and neutron transport, e.g., nuclear reactor design and shielding (LEWIS AND MiLlER, 1984; MARCHUK AND LEBEDEV, 1986). In addition, similar physical interpretations of the method in the kinetic theory of gases became known as the discrete velocity gas model (BROADWELL, 1964; PlatKOWSKI AND IllnER, 1988) which later evolved into models for large scale simulation of fluids: the lattice-gas cellular automaton, and lattice Boltzmann models (Rothman AND ZALESKI, 1997).

Notwithstanding steady improvements made to the quadrature rule method since its genesis, applications to the radiative transfer equation in extended atmospheres proved to be unsatisfactory (CHAPMAN, 1966). The original method became slowly convergent (or computationally demanding in the case of the discrete ordinates method) when sharp angular gradients needed to be resolved by means of increasing the number of Gauss directions. This weakness prompted Hummer AND RYBICKI (1971) to devise a novel method using a change to cylindrical coordinates suitable for adapting the approximate solution to regions of sharp gradients, in particular at the outer boundary where the radiative intensity is sharply peaked. Several investigations on extended atmospheres followed (Hummer AND RYBICKI, 1975; KunAsz AND HuMMER, 1974). Though successful, one drawback of the method is that it was tightly tailored to the problem at hand and did not easily lend itself to extensions to apply to other versions of transport equations, in particular, conservation laws governing coupled fluid flow and radiation.

In view of its simplicity and generality, the discrete ordinates method turned into the workhorse computational method of neutron transport and thermal radiative transfer, and it assumed a central position in astrophysical modeling. However it was not without trouble; in neutron transport calculations, care had to be taken to avoid flux distortions known as ray effects (LATHROP, 1968, 1971; LEWIS AND MILLER, 1984). Whereas for radiative transfer in extended atmospheres, the difficulty of introducing spatially local angular adaptivity into the method hindered its capability to produce cost effective accurate solutions. Recently, subdomain decomposition modifications have been proposed to allow for a spatially varying choice of the number of angular directions (see BAL, 2000, for an application to neutron transport). Note though that the resulting method sacrifices simplicity and has not been applied to problems in curved domains.

The foregoing prompted the development of the finite element PSDG method advocated here (sec. 4). The method pays special attention to the approximation of the radiative intensity field with respect to the angular direction by constructing the solution directly onto the phase space (see MARTIN et al., 1981; MiLleR JR. et al., 
1973; MoRDANT, 1986, for a similar approach in neutron transport). Hence, it allows for the greatest degree of flexibility to adapt an approximate solution to the angular direction and configurational space simultaneously. Although finite element methods are successful in solving problems with a high degree of locality, generic radiative transport problems are non-local by virtue of scattering and non-local boundary conditions. Consequently, a conventional finite element implementation will lead to an intricate globally coupled algebraic problem. To circumvent global connectivity, an iterative approach (sec. 3), known in astrophysics as the Eddington factor iteration, is combined with the PSDG method (similar strategies have been used in neutron transport and heat transfer calculations CHUI AND RAITHBY, 1993; LEWIS AND MILLER, 1984; RAITHBY AND CHUi, 1990). The iterative approach uses the angular moments of the radiative transfer equation, namely, radiative energy and momentum equations, to help set the correct energy and pressure levels in the configurational domain.

For many years, the configurational space DG method has been used in conjunction with the discrete ordinates method in the area of neutron transport (LEWIS AND MILlER, 1984). In fact, the DG method was originally proposed for a neutrontransport-motivated problem (consult COCKBURN et al., 2000, for the DG method history and modern applications mostly in fluid flow calculations). When used in companion with the discrete ordinate method, the DG method only approximates the solution in the configurational space. The combination is particularly effective when a few angular directions suffice to capture mildly anisotropic radiation beams. However, highly anisotropic cases often require too many directions and the method becomes prohibitively expensive.

The PSDG method advanced here expands the strength of the spatial DG method to apply to the entire phase space, thereby eliminating the need for the discrete ordinate approximation of the angular direction. Hence, the accuracy of the approximate solution can be improved throughout the space while advantage can be taken of adaptive methods to significantly reduce the computational cost. The method promises to be extensible to substantially more complex models of coupled radiation and fluid flow as it employs a Galerkin-type weak formulation (sec. 4.1) similar to those used to solve hyperbolic conservation laws of fluid flow which form the basis of radiative hydrodynamics (Minalas And Weibel-Minalas, 1999). The PSDG method is applied to the hyperbolic partial differential form of the radiative transfer equation (3.1) for extended atmospheres since the integral (non-local) contribution in the equation is updated iteratively as an energy source term (3.1a) using moments (3.2) of the radiative transport equation. The solution iterates are constructed element by element, preserving the normal component of the phase-space flux of the specific intensity (4.3), and following the characteristic directions of the hyperbolic operator (which define computational wave fronts, sec.4.2) wherein computational parallelism can be exploited.

Oak Ridge National Laboratory Technical Report ORNL/TM-2003/072 pp. 1-44 
Four cases of increasing atmosphere diameter spanning three orders of magnitude were studied utilizing the PSDG method. In addition, many different intensities of scattering were investigated by varying the value of the scattering power law exponent (sec.6). The computational results (sec.6) obtained for all combination of parameters correctly reproduced the asymptotic values (sec.2.1 and 2.3) of key physical quantities in the diffusive and transparent limits. In addition, details of the formation of the forward peaking and diffusive-transparent transition regions are captured accurately and made vivid by means of a log-polar surface of the normalized specific radiation intensity field (fig. 6.5 and 6.4).

The computations were carried out in multiple computer architectures and computing times were compared. The calculation of moments of the intensity field accounted for a large fraction of the total time $(\approx 50 \%)$ even when efficiently performed (sec. 4.3). The resulting method proved to be robust, accurate, and fast. Typically thirteen iterations sufficed for a sublinear convergence (convergence order $\approx 0.7$, sec. 6.4).

Last but not least, a careful description of boundary conditions, in particular at the origin (sec. 2.2), is made in the light of algorithm design and physical meaning.

\section{The spherical-geometry transfer problem}

For the applications treated in this manuscript, the radiative specific intensity field $I:(\boldsymbol{x}, \boldsymbol{\xi}, \nu, t) \in \Omega \times \boldsymbol{V} \times \mathbb{P} \times \mathbb{P} \rightarrow \mathbb{P}$ is a non-negative mapping of the position $\boldsymbol{x}$ in the Euclidean manifold $\Omega$, direction $\boldsymbol{\xi}(\|\boldsymbol{\xi}\|=1)$ in the translation vector space $\boldsymbol{V}$, frequency $\nu$ (or energy $h \nu$ ), and time $t$ in the set of positive real numbers $\mathbb{P}$. The assumption of steady, spherically symmetric, and gray radiation field reduces the set of independent variables to $(r, \mu)$, where $r$ is the radial coordinate of the spherical coordinate system, and $\mu:=\cos \theta$ is the direction cosine of a photon traveling in the direction $\theta$ with respect to the radial position vector (fig. 2.1). Occasionally the angle $\theta$ is referred to as the photon polar angle, and should not be confused with the polar angle coordinate of the underlying coordinate system.

Under the following restrictions:

- radiative equilibrium (time-independent radiation on static matter),

- gray radiation,

- pure conservative scattering (no true absorption or thermal emission processes),

- power-law radial isotropic scattering coefficient,

- absence of external illumination, and

Oak Ridge National Laboratory Technical Report ORNL/TM-2003/072 pp. 1-44 


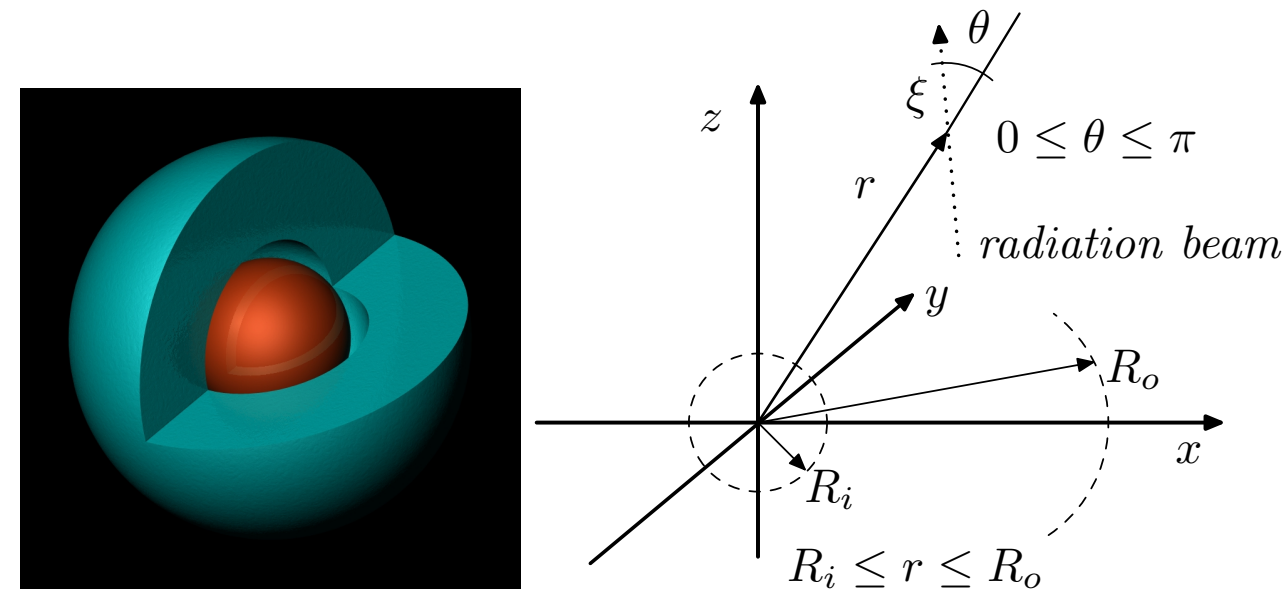

Fig. 2.1: Domain of the atmosphere. $R_{i}$ is the radius of the inner boundary placed into the diffusive core region. $\theta$ is the photon polar angle.

- nearly isotropic radiation deep into the stellar atmosphere,

the problem of radiation transfer reduces to: find the radiative specific intensity $I:(r, \mu) \in \Omega \rightarrow \mathbb{R}$ in the spherical annulus $\Omega:=\left\{(r, \mu) \in \mathbb{R}^{2} \mid R_{i} \leq r \leq R_{o}\right.$ and $-1 \leq \mu \leq+1\}$ satisfying,

$$
\mu \partial_{r} I+\frac{\left(1-\mu^{2}\right)}{r} \partial_{\mu} I=-k(r)\left(I-1 / 2 \int_{-1}^{+1} I\left(r, \mu^{\prime}\right) d \mu^{\prime}\right) \quad \text { in } \quad \Omega
$$

with outer and inner boundary conditions respectively,

$$
\begin{aligned}
& I\left(R_{o}, \mu\right)=0 \quad-1 \leq \mu \leq 0 \quad \text { and } \\
& I\left(R_{i}, \mu\right)=\gamma\left(L_{0}\right) I\left(R_{i},-\mu\right) \quad 0 \leq \mu \leq+1
\end{aligned}
$$

alternatively,

$$
I\left(R_{i}, \mu\right)=\beta\left(L_{0}\right) / \pi \quad 0 \leq \mu \leq+1
$$

where $k(r):=\alpha r^{-n}(n>1)$ is the scattering coefficient (its reciprocal is the local photon mean free path), and $\gamma\left(L_{0}\right)$ and $\beta\left(L_{0}\right)$ are real positive functions of the constant photon luminosity $L_{0}$. The outer boundary condition (2.1b) asserts that no radiation falls onto the atmosphere while at the inner boundary, (2.1c) states that the outward going radiation is a partial reflection of the incoming radiation, and the 
VALMOR DE AlmeidA

alternative boundary condition $2.1 \mathrm{~d}$ ) specifies that radiation at every outward pointing direction be a constant (comments on (2.1c) and (2.1d) follow in sec. 2.2). The aforementioned problem is non-local in view of the integral operator in (2.1a) and the inner boundary condition involving $\gamma$ and $\beta$ (sec. 2.2). Given an explicit form of $\gamma$ and $\beta$, 2.1) defines an unambiguous mathematical problem with an unique solution (not available in terms of tabulated functions).

\subsection{Asymptotic analytical results}

It is instructive to organize the asymptotic analytical results in a progressive sequence according to physical quantities derived from the radiation specific intensity with increasing rank, that is, from scalar to tensor. It just so happens that mathematically, the sequence involves operations with higher order angular moments.

\subsubsection{Radiative equilibrium}

The radiative equilibrium condition implies that the energy density emitted by an element of mass in the atmosphere is exactly balanced by the energy density absorbed by that element. This condition is satisfied for the problem at hand since

$$
\underbrace{\int_{-1}^{1} k(r) E(r) d \mu^{\prime}}_{\text {emitted energy density }}-\underbrace{\frac{4 \pi}{c} \int_{-1}^{1} k(r) I\left(r, \mu^{\prime}\right) d \mu^{\prime}}_{\text {absorbed energy density }}=0
$$

where $c$ is the speed of light, and $E(r):=(2 \pi / c) \int_{-1}^{+1} I\left(r, \mu^{\prime}\right) d \mu^{\prime}$ is the radiative energy density.

\subsubsection{Luminosity}

Taking the zeroth moment of 2.1a) (i.e. angular integration) and using (2.2),

$$
\begin{aligned}
\int_{-1}^{1}\left(\mu \partial_{r} I+\frac{\left(1-\mu^{2}\right)}{r} \partial_{\mu} I\right) d \mu^{\prime} & =0 \\
d_{r} F-\frac{2 F}{r} & =0 \\
F(r) & =\frac{\text { constant }}{r^{2}},
\end{aligned}
$$

are obtained, where $F(r):=2 \pi \int_{-1}^{1} I\left(r, \mu^{\prime}\right) \mu^{\prime} d \mu^{\prime}$ is the radial component (the other two components are null) of the net rate of the radiative energy flux at $r$. Hence, after solving the radiative energy equation $(2.3)$, it is concluded that the energy flux is not 
a constant, but rather it decays with the square of the radius. This behavior arises from the increase of the area of the spherical shell traversed by the radiation at $r$. Nevertheless the rate of loss of energy of the spherical shell, defined as the photon luminosity originated at $r$, is a constant $L_{0}$; thus

$$
L(r):=4 \pi r^{2} F(r)=L_{0} .
$$

\subsubsection{Radiative Stress}

Taking the first moment of (2.1a)

$$
\int_{-1}^{1}\left(\mu \partial_{r} I+\frac{\left(1-\mu^{2}\right)}{r} \partial_{\mu} I\right) \mu^{\prime} d \mu^{\prime}=\int_{-1}^{1}\left(-k(r)\left(I-\frac{1}{2} \int_{-1}^{1} I d \mu^{\prime \prime}\right)\right) \mu^{\prime} d \mu^{\prime},
$$

then

$$
\underbrace{d_{r} P+\frac{3 P-E(r)}{r}}_{(\operatorname{div} \boldsymbol{T})_{r}}=\frac{-k(r)}{c} F(r),
$$

where $P(r):=(2 \pi / c) \int_{-1}^{+1} I\left(r, \mu^{\prime}\right) \mu^{\prime 2} d \mu^{\prime}$. The result 2.6) is an equation referred to as the radial component of the radiative momentum. The radiative stress tensor $\boldsymbol{T}(r):=(1 / c) \int_{0}^{4 \pi} I(r, \mu) \boldsymbol{\xi} \otimes \boldsymbol{\xi} d w$, where $d w=\sin \theta d \theta=-d \mu$ is the element of solid angle about the pencil of radiation, has the following components relative to the orthonormal vector basis, $\left\{\boldsymbol{e}_{r}, \boldsymbol{e}_{\theta}, \boldsymbol{e}_{\phi}\right\}$ induced by the spherical coordinate system,

$$
\underbrace{\left(\begin{array}{ccc}
P & 0 & 0 \\
0 & P & 0 \\
0 & 0 & P
\end{array}\right)}_{\text {isotropic } P \boldsymbol{I}}+\frac{1}{2} \underbrace{\left(\begin{array}{ccc}
0 & 0 & 0 \\
0 & E-3 P & 0 \\
0 & 0 & E-3 P
\end{array}\right)}_{\text {deviatoric }}=\frac{1}{2}\left(\begin{array}{ccc}
2 P & 0 & 0 \\
0 & E-P & 0 \\
0 & 0 & E-P
\end{array}\right) \text {. }
$$

Hence, $P(r)$ is the radiation pressure at $r$ as indicated by the isotropic part of $\boldsymbol{T}$. Moreover, the principal directions of stress (eigenvectors) are $\left(\boldsymbol{e}_{r}, \boldsymbol{e}_{\theta}, \boldsymbol{e}_{\phi}\right)$. These are directions of largest traction wherein the shear contribution is null. The corresponding principal stresses (eigenvalues) are

$$
\lambda_{1}=P, \quad \text { and } \quad \lambda_{2}=\lambda_{3}=(E-P) / 2 .
$$

These are the magnitude of the traction in the principal directions. Finally, the principal invariants of the stress tensor result in,

$$
\begin{aligned}
\operatorname{tr} \boldsymbol{T} & :=\lambda_{1}+\lambda_{2}+\lambda_{3} & =E, \\
\operatorname{tr} \operatorname{cof} \boldsymbol{T} & :=\lambda_{1} \lambda_{2}+\lambda_{1} \lambda_{3}+\lambda_{2} \lambda_{3} & =\frac{(3 P+E)}{4}(E-P), \\
\operatorname{det} \boldsymbol{T} & :=\lambda_{1} \lambda_{2} \lambda_{3} & =P\left(\frac{E-P}{2}\right)^{2} .
\end{aligned}
$$




\subsubsection{Diffusive limit}

There are two mathematically and physically relevant limits in the solution of (2.1). The first is the diffusive limit which takes place deeply into the optically thick atmosphere, $r \rightarrow 0$. Here the photon mean free path is small and the photons travel in random walk by virtue of copious collision. The specific intensity is nearly isotropic therefore the pressure-energy density ratio (Eddington factor) $\lim _{r \rightarrow 0} P / E=1 / 3$, and the state of stress (2.7) becomes isotropic $P \boldsymbol{I}=(E / 3) \boldsymbol{I}$ for vanishingly small $r$. Note that the energy density is equally partitioned along the principal directions of stress; hence the magnitude of the traction in each direction is $E / 3$. The asymptotic behavior of the energy density can be obtained from (2.6) using $P / E=1 / 3$ and $\lim _{r \rightarrow 0}(3 P-E) / r=0$, thus

$$
\lim _{r \rightarrow 0} E=\frac{3 \alpha L_{0}}{4 \pi c} \frac{r^{-(n+1)}}{(n+1)} \quad \text { and } \quad \lim _{r \rightarrow 0} P=\frac{\alpha L_{0}}{4 \pi c} \frac{r^{-(n+1)}}{(n+1)}
$$

In addition, the principal stresses $(2.8)$ and the stress invariants 2.9 become, respectively,

$$
\begin{gathered}
\lim _{r \rightarrow 0} \lambda_{1}=\lim _{r \rightarrow 0} \lambda_{2}=\lim _{r \rightarrow 0} \lambda_{3}=E / 3, \quad \text { and } \\
\lim _{r \rightarrow 0} \operatorname{tr} \boldsymbol{T}=E, \quad \lim _{r \rightarrow 0} \operatorname{tr} \operatorname{cof} \boldsymbol{T}=E^{2} / 3, \text { and } \quad \lim _{r \rightarrow 0} \operatorname{det} \boldsymbol{T}=(E / 3)^{3} .
\end{gathered}
$$

\subsubsection{Streaming limit}

The second notable limit in the solution of (2.1) is encountered at the surface of an extended atmosphere, $r \rightarrow \infty$, where the scattering coefficient is vanishingly small, $\lim _{r \rightarrow \infty} k(r)=0$. The solution of (2.1) in this limit is of the form,

$$
\lim _{r \rightarrow \infty} I(r, \mu)=\frac{I_{0}(r)}{2 \pi} \delta(\mu-1)
$$

referred to as the outward peaking of the radiation specific intensity at the outer boundary. This behavior stems from the fact that the outer shells of an extended atmosphere behave as a vacuum (in view of the inverse power law scattering assumption) and the radiation becomes highly anisotropic in the absence of scattering (the photon mean free path is large and photons travel without collision). Thus, the radiation beam tends to align perfectly with the radial coordinate direction. However, because the luminosity is a constant and the area of the outer surface is very large, the magnitude of the delta peak, $I_{0}$, is very small. That is, the asymptotic value of 
$I_{0}$ follows from the luminosity:

$$
\begin{aligned}
\lim _{r \rightarrow \infty} F(r, \mu) & :=2 \pi \int_{-1}^{1} \lim _{r \rightarrow \infty} I\left(r, \mu^{\prime}\right) \mu^{\prime} d \mu^{\prime} \\
& =I_{0}(r) \text { by 2.10, }
\end{aligned}
$$

thus by 2.5

$$
I_{0}(r)=L_{0} / 4 \pi r^{2}
$$

Hence, the forward peak is a qualitative behavior of $I$ that requires a high resolution method for computing an accurate quantitative representation of a delta-like function of vanishingly small magnitude. In view of (2.10), the following limits are expected for problem (2.1)

$$
\begin{aligned}
\lim _{r \rightarrow \infty} I(r, \mu) & =\frac{L_{0}}{4 \pi r^{2}} \delta(\mu-1) & & \text { by 2.11, } \\
\max \lim _{r \rightarrow \infty} I(r, \mu) & =\frac{L_{0}}{4 \pi r^{2}} & & \text { from 2.10, } \\
\lim _{r \rightarrow \infty} c E(r) & =\lim _{r \rightarrow \infty} F(r)=\lim _{r \rightarrow \infty} c P(r) & & \text { from 2.10, } \\
\lim _{r \rightarrow \infty} c E(r) 4 \pi r^{2} & =\lim _{r \rightarrow \infty} c P(r) 4 \pi r^{2}=L_{0} & & \text { by 2.5 and 2.12. }
\end{aligned}
$$

From 2.13 the ratio of pressure to energy tends to unity, $P / E \rightarrow 1$, and the state of stress (2.7) becomes highly anisotropic with components, with respect to the basis $\left\{\boldsymbol{e}_{r}, \boldsymbol{e}_{\theta}, \boldsymbol{e}_{\phi}\right\}$, equal to

$$
\left(\begin{array}{lll}
P & 0 & 0 \\
0 & 0 & 0 \\
0 & 0 & 0
\end{array}\right) .
$$

In the streaming limit, the eigenvalues (2.8) and invariants (2.9), respectively,

$$
\begin{aligned}
& \lim _{r \rightarrow \infty} \lambda_{1}=E, \quad \text { and } \quad \lim _{r \rightarrow \infty} \lambda_{2}=\lim _{r \rightarrow \infty} \lambda_{3}=0, \quad \text { and } \\
& \lim _{r \rightarrow \infty} \operatorname{tr} \boldsymbol{T}=E, \quad \lim _{r \rightarrow \infty} \operatorname{tr} \operatorname{cof} \boldsymbol{T}=0, \quad \lim _{r \rightarrow \infty} \operatorname{det} \boldsymbol{T}=0,
\end{aligned}
$$

show that the energy density is no longer equally partitioned among the principal directions of stress, and that the stress tensor is singular. At the outer boundary of an extended atmosphere the radiation traction exerted on the stellar medium is null in all but one direction, the outward pointing radial principal direction. Because the radial direction is an eigenvector of the stress tensor the traction vector points in the same direction, and its magnitude is $E$; the single nonzero eigenvalue $\lambda_{1}$. 


\subsection{The inner boundary condition}

There are alternative ways to enforce the diffusive limit at the inner radius of problem (2.1); that are not all equivalent. In general, different choices of boundary condition lead to small differences in the solution near the origin. Other choices can introduce quantitative and qualitative errors in the solution that may or may not disappear with increasing radius. Of concern is the performance of a particular algorithm that can be adversely affected by an unsuitable choice of boundary condition, for this reason a "good" choice is at least of practical importance.

The present choices (2.1c)- $2.1 \mathrm{~d}$ ) were found to be suitable and convenient to constructing an approximate solution via a discontinuous Galerkin method (sec. 2.1.4). The condition (2.1c) introduces a finite discontinuity in $I\left(R_{i}, 0\right)$ at the zero polar angle which is readily captured by the solution method. The jump sets an energy flux, consistent with a given luminosity $L_{0}$, which should vary as in (2.5). The verification of (2.5) pointwise is an indication of the accuracy of the solution method (fig. 6.9).

The origin of the spherical domain in (2.1) is a point of singularity where the energy density, energy flux, and momentum flux grow without bounds (sec. 2.1.4). To avoid calculations with very large numbers, an inner boundary is created at a sufficiently small radius $R_{i}$. When $R_{i}$ is near the origin, $I\left(R_{i}, \mu\right)$ is nearly isotropic. This small departure from isotropy is critical to setting a finite energy flux emitted by the star.

Finding the coefficient $\gamma$ in the quasi-reflection condition implied by $2.1 \mathrm{c})$ requires the conservation of luminosity (2.5) and the fact that $\int_{-1}^{0} I\left(R_{i}, \mu^{\prime}\right) \mu^{\prime} d \mu^{\prime}=$ $-\int_{0}^{1} I\left(R_{i},-\mu^{\prime}\right) \mu^{\prime} d \mu^{\prime}$, with end result

$$
\gamma=1-\frac{2 L_{0}}{4 \pi R_{i}^{2} F_{i}^{-}}
$$

where $F_{i}^{-}:=2 \pi \int_{-1}^{0} I\left(R_{i}, \mu^{\prime}\right) \mu^{\prime} d \mu^{\prime}<0$ is the unknown incoming flux into the core. Therefore, $\gamma$ introduces a non-local boundary condition (in practical calculations $\gamma-1 \approx 0.4-0.5 \%$.

A partial isotropy argument in the outgoing direction $(0 \leq \mu \leq 1)$ was used in proposing (2.1d) since $\beta$ is independent of $\mu$. Similarly, as in the previous derivation for $\gamma$, the actual form of $\beta$ was obtained from the conservation of luminosity (2.5). Therefore, from (2.1c),

$$
\begin{aligned}
& \beta=\underbrace{F_{i}}_{\text {net flux at } R_{i}}-\underbrace{F_{i}^{-}}_{\text {incoming flux at } R_{i}} \\
& \beta=\frac{L_{0}}{4 \pi R_{i}^{2}}-2 \pi \int_{-1}^{0} I\left(R_{i}, \mu^{\prime}\right) \mu^{\prime} d \mu^{\prime},
\end{aligned}
$$


which shows that the boundary condition (2.1d) is non-local.

It remains to show that these choices of $\gamma$ and $\beta$ will lead to the diffusive limit asymptotics (sec. 2.1.4). To that end, if one observes that the incoming radiation at the inner radius $I(0,-1 \leq \mu \leq 0)$ is sufficiently flat with respect to $\mu$, the limit $P / E \rightarrow 1 / 3$ for the energy-to-pressure ratio follows; so do the results in section 2.1.4.

\subsection{Scaling and working formulae}

The choice of units of length and energy can simplify the aforementioned formulae making them suitable for a comprehensive parameter analysis. Let the units of length, energy flux and specific radiation intensity, and energy density be

$$
\alpha^{\frac{1}{n-1}}, \quad \frac{L_{0}}{4 \pi \alpha^{\frac{2}{n-1}}}, \text { and } \frac{L_{0}}{4 \pi \alpha^{\frac{2}{n-1}} c},
$$

respectively. The scaled quantities measured in these units will be denoted by the same variables used earlier to avoid cumbersome notation. That is, one would indicate the newly scaled quantity by a hat superscript, e.g.

$$
r=\widehat{r} \alpha^{\frac{1}{n-1}}, \quad I=\widehat{I} \frac{L_{0}}{4 \pi \alpha^{\frac{2}{n-1}}}, \quad F=\widehat{F} \frac{L_{0}}{4 \pi \alpha^{\frac{2}{n-1}}}, \quad E=\widehat{E} \frac{L_{0}}{4 \pi \alpha^{\frac{2}{n-1}} c}, \text { etc. }
$$

Then, after rewriting the formulae in terms of the hat quantities, the superscript is removed. The results follow in the ensuing sections.

\subsubsection{Transfer problem}

Problem (2.1) remains formally unchanged in the new scaled version but with the following new definition

$$
k(r):=r^{-n}, \quad \gamma:=1-\frac{1}{R_{i}^{2} F_{i}^{-}}, \quad \beta:=R_{i}^{-2}-F_{i}^{-} .
$$

From the first identity above, the local mean free path of a photon emitted at radius $r$ is $k^{-1}(r)=r^{n}$ measured in units of $\alpha^{\frac{1}{n-1}}$. The effect of varying the power law exponent $n$ is deduced from this result: photons emitted in the region $r<1$ possess mean free paths smaller than the radius they are emitted at, decreasing with increasing $n$, while photons emitted in the region $r>1$ have mean free paths greater than the radius, increasing with $n$. Regardless the value of $n$, the mean free path (in units of $\alpha^{\frac{1}{n-1}}$ ) of a photon emitted at $r=1$ is equal to the unit. The effect of increasing $n$ is to make the diffusive region more diffusive, and the transparent regions more transparent. 


\subsubsection{Luminosity and stress}

The luminosity conservation in units of (2.16) reduces to

$$
r^{2} F(r)=1
$$

and the momentum equation to

$$
d_{r} P+\frac{3 P-E(r)}{r}=-k(r) F(r),
$$

with pressure, stress, and principal stresses measured in units of energy density, and principal invariants measured in the corresponding powers of the same unit; all formulae related to stress remain formally unchanged.

It is instructive to report results on the "undiluted" quantities, namely,

$$
\begin{aligned}
\mathscr{E}(r) & :=r^{2} E(r), \quad \mathscr{F}(r):=r^{2} F(r)=1, \\
\mathscr{P}(r) & :=r^{2} P(r), \text { and } \quad \operatorname{det} \mathscr{T}(r):=\left(r^{2}\right)^{3} \operatorname{det} \boldsymbol{T} .
\end{aligned}
$$

That is, the factor $r^{2}$ tends to offset the natural dilution of the fields due to the increase in the area of the spherical shell at $r$.

\subsubsection{Streaming limit}

The asymptotic magnitude of the forward peak in units of $L_{0} /\left(4 \pi \alpha^{\frac{2}{n-1}}\right)$ is

$$
\max \lim _{r \rightarrow \infty} I(r, \mu)=r^{-2},
$$

while energy and pressure expressed in units of $L_{0} /\left(4 \pi \alpha^{\frac{2}{n-1}} c\right)$ turn into a similar result,

$$
\begin{aligned}
\lim _{r \rightarrow \infty} E(r) & =\lim _{r \rightarrow \infty} P(r)=r^{-2}, \quad \lim _{r \rightarrow \infty} \operatorname{det} \boldsymbol{T}=0 \\
\lim _{r \rightarrow \infty} \mathscr{E}(r) & =\lim _{r \rightarrow \infty} \mathscr{P}(r)=1, \text { and } \quad \lim _{r \rightarrow \infty} \operatorname{det} \mathscr{T}=0 .
\end{aligned}
$$

\subsubsection{Diffusive limit}

Near the core $r \rightarrow 0$, energy and pressure limits result in,

$$
\begin{array}{llrl}
\lim _{r \rightarrow 0} E(r)=\frac{3 r^{-(n+1)}}{n+1}, & \lim _{r \rightarrow 0} P(r) & =\frac{r^{-(n+1)}}{n+1}, & \lim _{r \rightarrow 0} \operatorname{det} \boldsymbol{T}(r)=(E / 3)^{3} \\
\lim _{r \rightarrow 0} \mathscr{E}(r)=\frac{3 r^{-n+1}}{n+1}, & \lim _{r \rightarrow 0} \mathscr{P}(r)=\frac{r^{-n+1}}{n+1}, & \text { and } & \lim _{r \rightarrow 0} \operatorname{det} \mathscr{T}(r)=(\mathscr{E} / 3)^{3} .
\end{array}
$$




\subsubsection{Radial optical depth}

It is often more important to measure distance from the outer surface of the atmosphere towards the interior. The radial optical depth is such a measure in units of photon mean free path defined as,

$$
\tau(r):=\underbrace{\int_{R_{o}}^{r} k(r) d r}_{\text {unscaled }}=\underbrace{\int_{R_{o}}^{r} r^{-n} d r}_{\text {scaled }}=\frac{r^{-(n-1)}-R_{o}^{-(n-1)}}{n-1} .
$$

Thus $\tau(r)$ is the number of mean free paths between the outer boundary and the radial position $r$ measured along the radius. The optical depth of the origin is $\tau(0)=\infty$ which renders the atmosphere of the transfer problem (2.1) infinitely optically thick. Another key value is $\tau\left(r_{t}\right)=1$, that is, the radial position $r_{t}$ exactly one mean free path away from the outer boundary. From (2.21),

$$
r_{t}=\frac{1}{\left(n-1+R_{o}^{-(n-1)}\right)^{\frac{1}{n-1}}}<1 .
$$

Therefore photons emitted at optical depths equal or less than one, measured from the surface of the atmosphere, stream to the outer boundary without collision since the distance they travel is less or equal to one mean free path. On the other hand, photons emitted at optical depths greater than one undergo an increasing number of collisions with increasing optical depth, and they tend to become trapped in the diffusive region. Therefore the radial position $r_{t}$ defines the diffusive-transparent transition region.

Henceforth all quantities are assumed scaled.

\section{Radiative pressure iteration}

A natural scheme for solving the integrodifferential problem (2.1) can be devised in two steps. First, assume the integral operator as a given function of $r$ and solve the resulting hyperbolic partial differential equation in phase space. Second, iterate to correct the scattering integral. This is in essence a fixed-point method likely to fail when scattering becomes dominant. An attemptable improvement is to use the moments of the original integral differential equation to perform the necessary integral correction; that is, to help to set the correct energy level throughout the system. One successful scheme, often referred to as the Eddington iteration method (HUMMER AND RYBICKI, 1971; Minalas AND WeiBel-Minalas, 1999), uses the pressure-to-energy ratio as a relaxation/corrective parameter to iterate between the radiative transfer 
equation and its zeroth and first moment equations (radiative energy and momentum equations). This improvement is explored here (algorithm 3.1) in conjunction with a discontinuous Galerkin method.

The hyperbolic problem (scaled as in sec. 2.3) corresponding to (2.1) at the $k$ th iteration is

$$
\mu \partial_{r} I^{(k)}+\frac{\left(1-\mu^{2}\right)}{r} \partial_{\mu} I^{(k)}=-r^{-n}\left(I^{(k)}-E(r)^{(k-1)} / 4 \pi\right) \quad \text { in } \quad \Omega
$$

with inner and outer boundary conditions respectively

$$
\begin{aligned}
& I\left(R_{o}, \mu\right)^{(k)}=0 \quad-1 \leq \mu \leq 0 \quad \text { and } \\
& I\left(R_{i}, \mu\right)^{(k)}=\gamma^{(k-1)} I\left(R_{i},-\mu\right)^{(k-1)} \quad 0 \leq \mu \leq+1,
\end{aligned}
$$

where $\gamma^{(k-1)}=1-1 /\left(R_{i}^{2}\left(F_{i}^{-}\right)^{(k-1)}\right)$ while the radiative momentum and energy equations, 2.19) and (2.18) are combined into one for the undiluted pressure $\mathscr{P}: r \in$ $\left[R_{i}, R_{o}\right] \rightarrow \mathbb{R}$

$$
\left.d_{r} \mathscr{P}^{(k)}+\left(1-\frac{1}{f(r)^{(k)}}\right) \frac{\mathscr{P}^{(k)}}{r}=-r^{-n} \quad \text { in } \quad\right] R_{i}, R_{o}[,
$$

with boundary condition at the surface

$$
\mathscr{P}\left(R_{o}\right)^{(k)}=\frac{f\left(R_{o}\right)^{(k)}}{g^{(k)}}
$$

where $f(r)^{(k)}:=P(r)^{(k)} / E(r)^{(k)}$ is the pressure-to-energy ratio known as the Eddington factor which acts as a relaxation field, and $g^{(k)}:=F\left(R_{o}\right)^{(k)} / E\left(R_{o}\right)^{(k)}$.

Note that from (2.18), this ratio equals the undiluted pressure when the value of $f\left(R_{o}\right)^{(k)}$ is known correctly (converged). The foregoing prompts the iterative algorithm (3.1) that explains the use of the Eddington factor. The solution method starts by solving the momentum equation (3.2) so the resulting energy distribution is inserted in (3.1). For this reason, an initial approximation for the pressure-to-energy ratio is required. Because the Eddington factor varies from $1 / 3$ in the diffusive limit, to 1 in the free streaming limit (sec. 2.1), a reasonable choice is $f(r)^{(0)}=1-\frac{2}{3} e^{-r / r_{t}}$ (Hummer AND RYBICKI, 1971). A choice for $g^{(0)}$ is also necessary; hence, in view of its significance a value between 1 and $1 / 2$ is appropriate.

Algorithm 3.1. Radiative pressure discontinuous Galerkin iteration

1. Set $f(r)^{(0)}=1-\frac{2}{3} e^{-r / r_{t}}$, and $g=1 / 2$ 
2. Solve 3.2 for $\mathscr{P}(r)^{(0)}$ (sec. 5)

3. Set $I(r, \mu)^{(0)}=0$, and override $3.1 \mathrm{c}$ with $I\left(R_{i}, 0 \leq \mu \leq 1\right)^{(0)}=1$

4. Set $E(r)^{(0)}=\mathscr{P}(r)^{(0)} / r^{2} f(r)^{(0)}$

5. Set tol $=10^{-7}$

6. Set $k=1 ; N_{\max }=15 ;$ converged $=0$

7. While $k \leq N_{\max }$ and !converged Do:

8. Solve (3.1) for $I(r, \mu)^{(k)}$ (algorithm 4.1)

9. $\quad$ Compute elemental relative update norm $\left\|\delta I_{1}^{(k)} / I^{(k)}\right\|_{\mathrm{H}^{1}\left(\mathscr{K}_{e}\right)} \forall \mathscr{K}_{e}$

10. Compute max. DG residual $\max _{\mathscr{K}_{e} \in \mathscr{T}_{h}}\left({ }^{e} b_{i}^{(k)} /{ }^{e} b_{i}^{(1)}\right)$ 4.14

11. If $\max _{\mathscr{K}_{e} \in \mathscr{T}^{h}}\left\|\delta I_{1}^{(k)} / I^{(k)}\right\|_{\mathrm{H}^{1}\left(\mathscr{K}_{e}\right)} \geq$ tol then

12. Compute $f(r)^{(k)}=P(r)^{(k)} / E(r)^{(k)}$ from $I(r, \mu)^{(k)}$

13. Compute $g=F\left(R_{o}\right)^{(k)} / E\left(R_{o}\right)^{(k)}$ from $I\left(R_{o}, \mu\right)^{(k)}$

14. Solve 3.2 for $\mathscr{P}(r)^{(k)}$ (sec. 5)

15. $\quad$ Compute $E(r)^{(k)}=\mathscr{P}(r)^{(k)} / r^{2} f(r)^{(k)}$

16. Compute $\left(F_{i}^{-}\right)^{(k)}:=\int_{-1}^{0} I\left(R_{i}, \mu^{\prime}\right)^{(k)} \mu^{\prime} d \mu^{\prime}$ and obtain $\gamma^{(k)}$ 2.17b)

17. Else

18. $\quad$ converged $=k$

19. Endif

20. EndWhileDo

Steps 8-13 are elaborated in section 4, and steps 14-15, in section 5. Noteworthy is step 15 which sets the energy level throughout the space, providing the correction on the right side of (3.1a). In practice, this algorithm is found to be convergent and robust for a wide range of parameters (sec. 6).

\section{The homogeneous problem}

At the $k$ th iteration, problem (3.1) is linear hyperbolic with known characteristic curves. The Dirichlet boundary conditions are applied to the part of the boundary where the angle between the outward pointing normal and the tangent to the characteristic curves intersecting the boundary is greater than $\pi / 2$. Only the segments of the boundary that satisfy this condition allow prescription of data. An approximate solution for this sort of problem can be obtained quickly, accurately, and economically (reduced computational storage) by a finite element discontinuous Galerkin method. To simplify the description of the solution method, the superscript $k$ is dropped, $E(r)^{(k-1)} / 4 \pi$ is denoted $J(r)$, and the boundary condition (3.1c) is rewritten as $I\left(R_{i}, \mu\right)=A(\mu)$ with $A$ representing a known function. 
Let $\boldsymbol{v}:(r, \mu) \in \Omega \rightarrow \mathbb{R}^{2}$ be the tangential vector field to the characteristic lines in (3.1) (fig. 4.1). The components of this vector field with respect to the orthonormal basis $\left\{\boldsymbol{i}_{r}, \boldsymbol{i}_{\mu}\right\}$ induced by the phase space coordinate system are $\left(\mu,\left(1-\mu^{2}\right) / r\right)$. Let the radiation inflow boundary be denoted by $\partial \Omega^{-}:=\{(r, \mu) \in \partial \Omega \mid \boldsymbol{v} \cdot \boldsymbol{n}<0\}$, with $\boldsymbol{n}$ denoting the unit outward pointing normal on $\partial \Omega$. Then the boundary conditions (3.1b) and (3.1c) can be expressed compactly as $I \equiv I_{b^{-}}$on $\partial \Omega^{-}$. Define $I \equiv I^{*}+\delta I$ where $I^{*}$ is some convenient extension of the data $I_{b^{-}}$onto $\Omega$, therefore by construction $\left.\delta I\right|_{\partial \Omega^{-}} \equiv 0$, and problem (3.1) for $\delta I$ is homogeneous

$$
\begin{aligned}
\nabla \delta I \cdot \boldsymbol{v}+r^{-n} \delta I & =-S^{*} \quad \text { in } \quad \Omega, \\
\delta I & =0 \quad \text { on } \partial \Omega^{-},
\end{aligned}
$$

where the residual $S^{*}(r, \mu):=\nabla I^{*} \cdot \boldsymbol{v}+r^{-n}\left(I^{*}-J\right)$ should be square integrable in $\Omega$; this requires continuity of $I^{*}$ in the $\boldsymbol{v}$ direction. The phase space gradient operator is denoted $\nabla(\cdot):=\mu(\cdot) \boldsymbol{i}_{r}+\left(1-\mu^{2}\right) / r(\cdot) \boldsymbol{i}_{\mu}$. Thus, given any suitable function $I^{*}$, the solution of (4.1) delivers the solution of (3.1) via $I \equiv I^{*}+\delta I$.

When $k=1$ (algorithm 3.1, step 6) $I^{*}$ is set as in (4.7) (at this point its value is not relevant), and $J(r)=E(r)^{(0)} / 4 \pi$ (from step 4); together they define $S^{*}(r, \mu)$. The solution of (4.1), $\delta I_{1}^{(1)}$, is the correction to $I^{*}$ that provides the new radiative intensity field iterate $I_{1}^{(1)} \equiv I^{*}+\delta I_{1}^{(1)}$. In practice, to be sure that the linear problem (4.1) has been solved to a satisfactory accuracy, a second "inner" iteration is performed by setting $I^{*} \leftarrow I_{1}^{(1)}$, recomputing $S^{*}$, and solving (4.1) for $\delta I_{2}^{(1)}$. After the update $I_{2}^{(1)} \equiv I^{*}+\delta I_{2}^{(1)}$, the first iterate has been refined, and an indication of whether the solution method used is correct is obtained from some measure of the magnitude of the correction which should be vanishingly small, say $\left\|\delta I_{2}^{(1)} / I_{2}^{(1)}\right\| \approx 0$ (typically $10^{-14}-10^{-15}$, fig. 6.12).

At the $k$ th iteration, $I^{*} \equiv I^{(k-1)}$ and $A(\mu)=\gamma^{(k-1)} I\left(R_{i},-\mu\right)^{(k-1)}$, and the solution of (4.1) is repeated for $\delta I_{1}^{(k)}$ and $\delta I_{2}^{(k)}$. Note that the second update must be always vanishingly small, therefore the first update provides a measure of the convergence of the iterative method for solving the original problem (2.1). In typical computations when $\left\|\delta I_{1}^{(k)} / I_{1}^{(k)}\right\|<10^{-7}$ the iteration is stopped (steps 11 and 18, algorithm 3.1). The particular norm used depends on the method used to solve (4.1) as described in the next subsection.

Finally, when $\left\|\delta I_{1}^{(k)} / I_{1}^{(k)}\right\| \approx 0$ then, from 4.1a), the residual norm $\left\|S^{*}\right\| \approx 0$, and $I^{*} \equiv I^{(k-1)} \approx I^{(k)}$ is an approximate solution of (2.1). Note that in algorithm (3.1) the norm of the residual (step 10) is not used to stop the iterations. This is so because unlike the relative update norm, it is in general difficult to scale the residual so that an indication of convergence is associated to a small residual norm. Hence the relative residual norm $\left\|S^{(k)}\right\| /\left\|S^{(1)}\right\|$ (the residual reduction since the first iteration) 


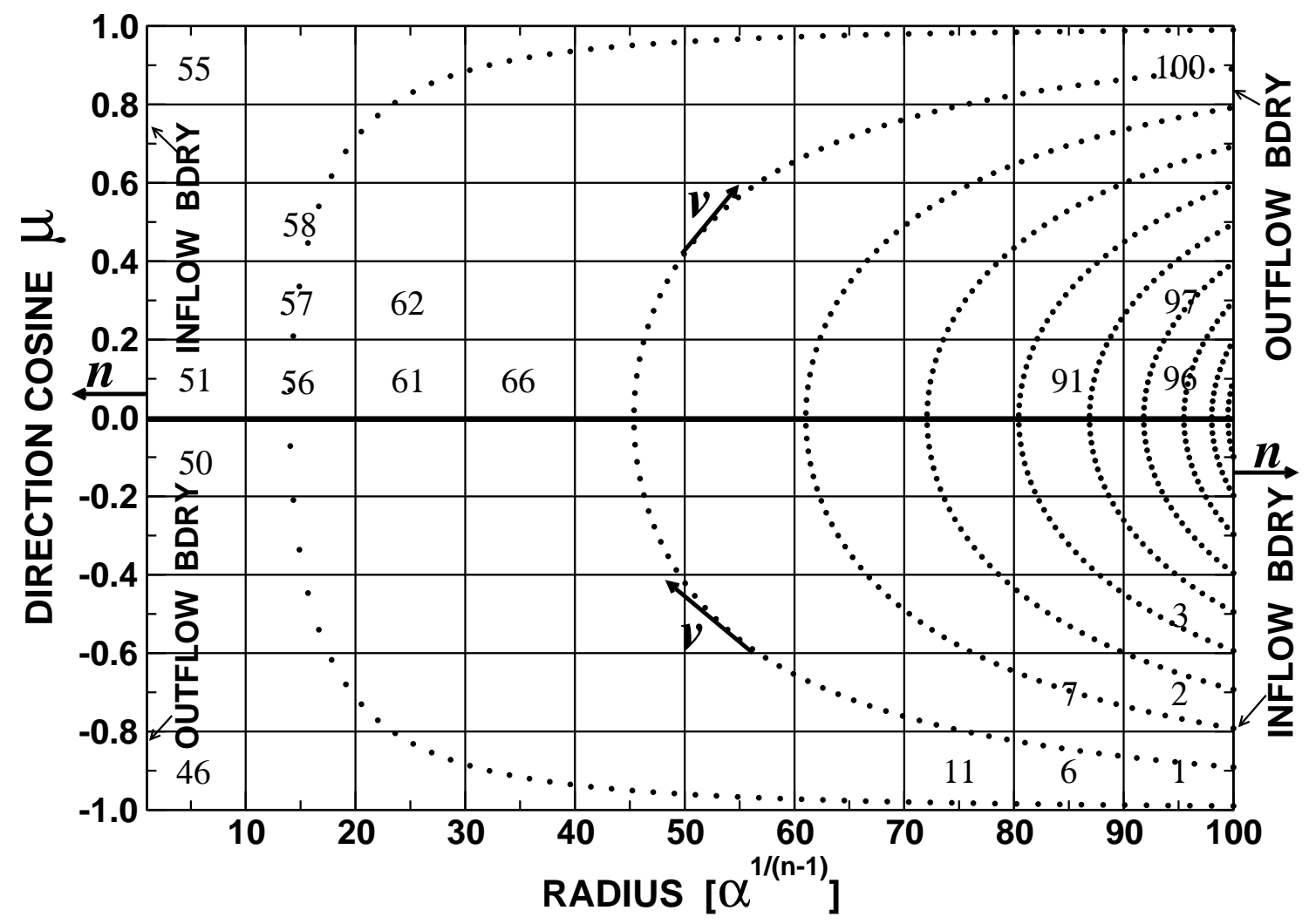

Fig. 4.1: Phase space characteristic lines. These lines are tangent to the vector field $\boldsymbol{v}=\mu \boldsymbol{i}_{r}+\left(1-\mu^{2}\right) / r \boldsymbol{i}_{\mu}$, therefore they are computed by numerical integration of $\dot{r}(\sigma) \boldsymbol{i}_{r}+\dot{\mu}(\sigma) \boldsymbol{i}_{\mu}=\boldsymbol{v}$. The combination of the inflow segments form $\partial \Omega^{-}$where $\boldsymbol{v} \cdot \boldsymbol{n}<0$. Numbering within the grid illustrates the wave front finite element ordering (sec. 4.2). 
is observed, which typically is on the order of $10^{-10}$ at convergence (fig. 6.12), but not used as an indicator of convergence.

\subsection{A discontinuous Galerkin finite element method}

The method of choice for solving the homogeneous problem (4.1) is described next. Consider the weak formulation: find $\delta I \in \mathscr{S}(\Omega):=\left\{\delta I \in \mathrm{L}_{0}^{2}(\Omega) \mid \nabla \delta I \cdot \boldsymbol{v} \in \mathrm{L}^{2}(\Omega)\right\}$ such that

$$
(\nabla \delta I \cdot \boldsymbol{v}, \phi)+\left(r^{-n} \delta I, \phi\right)=-\left(S^{*}, \phi\right) \quad \forall \quad \phi \in \mathrm{L}_{0}^{2}(\Omega),
$$

where $(a, b):=\int_{\Omega} a b d \Omega$ is the inner product in $\mathrm{L}^{2}(\Omega), \mathrm{L}_{0}^{2}(\Omega):=\left\{\phi \in \mathrm{L}^{2}(\Omega) \mid \phi \equiv\right.$ 0 on $\left.\partial \Omega^{-}\right\}$is a linear space of square integrable functions with homogeneous data on the inflow boundary of $\Omega$, and the residual $S^{*} \in \mathrm{L}^{2}(\Omega)$. The trial functions $\delta I$ in the linear space $\mathscr{S}(\Omega)$ are required to be weakly continuous only along the $\boldsymbol{v}$ direction which is a sufficient request for regularity.

Let $\mathscr{T}^{h}:=\left\{\mathscr{K}_{e} \mid e=1, \ldots, N\right\}$ be a finite element partition of the polygonal domain $\Omega$, where $\bigcup_{e} \overline{\mathscr{K}}_{e}=\Omega, \bigcap_{e} \mathscr{K}_{e}=\emptyset$, and $\mathscr{K}_{e}$ is either a quadrilateral or a triangle for all $e$. Define the finite dimensional (finite element) linear functional space $\mathscr{X}_{0}^{h}(\Omega):=\left\{w \in \mathrm{L}_{0}^{2}(\Omega)|w|_{\mathscr{K}_{e}} \in \mathscr{P}_{k}\left(\mathscr{K}_{e}\right) \subset \mathrm{H}^{1}\left(\mathscr{K}_{e}\right) \forall \mathscr{K}_{e} \in \mathscr{T}^{h}\right\}$, where $\mathscr{P}_{k}\left(\mathscr{K}_{e}\right)$ is a space of (quasi) polynomials over $\mathscr{K}_{e}$ of degree $\leq k$. Note that $\mathscr{X}_{0}^{h}(\Omega)$ is not properly included in $\mathscr{S}(\Omega)$ but it has elements that can be used as trial functions in (4.2). The next theorem identify such elements.

Theorem 4.1. Let $\delta I^{h}$ denote elements in $\mathscr{X}_{0}^{h}(\Omega)$ such that the weighted-flux continuity condition is satisfied

$$
\left\langle\delta I_{+}^{h} \boldsymbol{v} \cdot \boldsymbol{n}, \phi\right\rangle_{\partial \mathscr{K}_{e}^{-}}=\left\langle\delta I_{-}^{h} \boldsymbol{v} \cdot \boldsymbol{n}, \phi\right\rangle_{\partial \mathscr{K}_{e}} \forall \phi \in \mathscr{X}_{0}^{h}(\Omega),
$$

where $\partial \mathscr{K}_{e}^{-}$is the subset of points of $\partial \mathscr{K}_{e}$ with inflow $\boldsymbol{v} \cdot \boldsymbol{n}<0$, and $\delta I_{-}^{h}$ represents the value of $\delta I^{h}$ outside $\mathscr{K}_{e}$ relative to $\boldsymbol{n}$ (the outward normal pointing vector on $\partial \mathscr{K}_{e}$ ). That is, $\delta I_{-}^{h}(\boldsymbol{y})=\lim _{s \rightarrow 0^{-}} \delta I^{h}(\boldsymbol{y}+s \boldsymbol{v}) \forall \boldsymbol{y} \in \partial \mathscr{K}_{e}^{-}$is the value outside $\mathscr{K}_{e}$, similarly $\delta I_{+}^{h}(\boldsymbol{y})=\lim _{s \rightarrow 0^{+}} \delta I^{h}(\boldsymbol{y}+s \boldsymbol{v})$ is the value inside. Also, the arclength integration on the boundary of $\mathscr{K}_{e}$ is denoted $\left\langle\delta I^{h} \boldsymbol{v} \cdot \boldsymbol{n}, \phi\right\rangle_{\partial \mathscr{K}_{e}}:=\int_{\partial \mathscr{K}_{e}} \delta I^{h} \boldsymbol{v} \cdot \boldsymbol{n} \phi d s$. Then, $\delta I^{h} \in \mathscr{S}(\Omega)$.

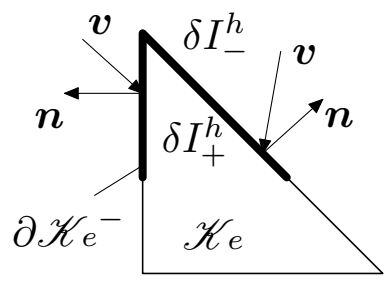


Proof. Since $\left.\delta I^{h}\right|_{\mathscr{K}_{e}} \in \mathrm{H}_{0}^{1}\left(\mathscr{K}_{e}\right) \forall \mathscr{K}_{e} \in \mathscr{T}^{h}$, then $\sum_{e=1}^{N} \int_{\mathscr{K}_{e}} \nabla \delta I^{h} \cdot \boldsymbol{v} d \Omega<\infty$. However

$$
\begin{aligned}
\sum_{e=1}^{N} \int_{\mathscr{K}_{e}} \nabla \delta I^{h} \cdot \boldsymbol{v} d \Omega & =\sum_{e=1}^{N}\left\{\int_{\partial \mathscr{K}_{e}} \delta I^{h} \boldsymbol{v} \cdot \boldsymbol{n} d s-\int_{\mathscr{K}_{e}} \delta I^{h} \operatorname{div} \boldsymbol{v} d \Omega\right\}<\infty, \\
& =\sum_{e=1}^{N}\left\{\int_{\partial \Omega \cap \partial \mathscr{K}_{e}} \delta I^{h} \boldsymbol{v} \cdot \boldsymbol{n} d s-\int_{\mathscr{K}_{e}} \delta I^{h} \operatorname{div} \boldsymbol{v} d \Omega\right\}<\infty, \quad \text { by }(4.3) \\
& =\int_{\partial \Omega} \delta I \boldsymbol{v} \cdot \boldsymbol{n} d s-\int_{\Omega} \delta I^{h} \operatorname{div} \boldsymbol{v} d \Omega<\infty, \\
& =\int_{\Omega} \nabla \delta I^{h} \cdot \boldsymbol{v} d \Omega<\infty,
\end{aligned}
$$

thus, $\nabla \delta I^{h} \cdot \boldsymbol{v} \in \mathrm{L}^{2}(\Omega)$ and $\delta I^{h} \in \mathscr{S}(\Omega)$.

Substituting a trial function $\delta I^{h}$ into 4.2 and selecting the test function $\phi$ conforming to $\mathscr{X}_{0}^{h}(\Omega) \subset \mathrm{L}_{0}^{2}(\Omega)$

$$
\sum_{e=1}^{N}\left(\nabla \delta I^{h} \cdot \boldsymbol{v}, \phi\right)_{\mathscr{K}_{e}}+\left(r^{-n} \delta I^{h}, \phi\right)_{\mathscr{K}_{e}}=-\sum_{e=1}^{N}\left(S^{*}, \phi\right)_{\mathscr{K}_{e}} \quad \forall \quad \phi \in \mathscr{X}_{0}^{h} \Omega,
$$

with $(a, b)_{\mathscr{K}_{e}}:=\int_{\mathscr{K}_{e}} a b d \Omega$, and integrating by parts the first term on the left side for each element $\mathscr{K}_{e}$, with its boundary oriented by its corresponding outward pointing normal vector $\boldsymbol{n}$, one obtains $\left(\nabla \delta I^{h} \cdot \boldsymbol{v}, \phi\right)_{\mathscr{K}_{e}}=-\left(\delta I^{h}, \operatorname{div}(\phi \boldsymbol{v})\right)_{\mathscr{K}_{e}}+\left\langle\delta I^{h} \boldsymbol{v} \cdot \boldsymbol{n}, \phi\right\rangle_{\partial \mathscr{K}_{e}}$. Therefore 4.4 becomes

$$
\begin{aligned}
\sum_{e=1}^{N}-\left(\delta I^{h}, \operatorname{div}(\phi \boldsymbol{v})\right)_{\mathscr{K}_{e}}+\left\langle\delta I^{h} \boldsymbol{v} \cdot \boldsymbol{n}, \phi\right\rangle_{\partial \mathscr{K}_{e}}+ & \left(r^{-n} \delta I^{h}, \phi\right)_{\mathscr{K}_{e}}= \\
& -\sum_{e=1}^{N}\left(S^{*}, \phi\right)_{\mathscr{K}_{e}} \quad \forall \quad \phi \in \mathscr{X}_{0}^{h} \Omega .
\end{aligned}
$$

From the flux continuity condition (theorem 4.1 ), $\left\langle\delta I^{h} \boldsymbol{v} \cdot \boldsymbol{n}, \phi\right\rangle_{\partial \mathscr{K}_{e}}$ can be replaced by $\left\langle\delta I_{+}^{h} \boldsymbol{v} \cdot \boldsymbol{n}, \phi\right\rangle_{\partial \mathscr{K}_{e}^{+}}+\left\langle\delta I_{-}^{h} \boldsymbol{v} \cdot \boldsymbol{n}, \phi\right\rangle_{\partial \mathscr{K}_{e}^{-}}$, where $\partial \mathscr{K}_{e}^{+}$is the subset of points of $\partial \mathscr{K}_{e}$ with outflow $\boldsymbol{v} \cdot \boldsymbol{n}>0$. The result is

$$
\begin{aligned}
\sum_{e=1}^{N}\left(\nabla \delta I^{h} \cdot \boldsymbol{v}, \phi\right)_{\mathscr{K}_{e}}+\left(r^{-n} \delta I^{h}, \phi\right)_{\mathscr{K}_{e}}-\left\langle\llbracket \delta I^{h} \rrbracket \boldsymbol{v} \cdot \boldsymbol{n}, \phi\right\rangle_{\partial \mathscr{K}_{e}^{-}} & = \\
& -\sum_{e=1}^{N}\left(S^{*}, \phi\right)_{\mathscr{K}_{e}} \quad \forall \quad \phi \in \mathscr{X}_{0}^{h}(\Omega),
\end{aligned}
$$


where $\llbracket \delta I_{e} \rrbracket:=\delta I_{+}^{h}-\delta I_{-}^{h}$ is the jump on $\delta I^{h}$ across the inflow area (line) $\partial \mathscr{K}_{e}^{-}$. Comparing the above against (4.2), no continuity requirement is necessary except within an element $\mathscr{K}_{e}$. The small price paid for recovering the solution of the original formulation in a full discontinuous framework is the appearance of the additional jump term. However as a bonus, this term enforces the conservation of the normal flux across element boundaries. Therefore, despite the non-divergence form of (4.6), the normal flux of the specific radiative intensity is conserved. In fact the jump term is the means for constructing approximate solutions in $\mathscr{S}(\Omega)$ using $\mathscr{X}_{0}^{h}(\Omega)$.

A similar rationality can be applied to the linear form $\left(S^{*}, \phi\right)_{\mathscr{K}_{e}}$. That is, it is desirable to build $S^{*}:=\nabla I^{*} \cdot \boldsymbol{v}+r^{-n}\left(I^{*}-J\right) \in \mathrm{L}^{2}(\Omega)$ from discontinuous pieces, $I^{* h} \in \mathscr{X}_{0}^{h}(\Omega)$, that form a valid $I^{*}$. For example, when $k=1$ (algorithm 3.1, step 7) $I^{*}$ is set as follows

$$
I^{*}(r, \mu)= \begin{cases}0 & \text { for } r=R_{o}, \text { and }-1 \leq \mu \leq 0, \\ 1 & \text { for } r=R_{i}, \text { and } 0 \leq \mu \leq+1, \\ 0 & \text { otherwise },\end{cases}
$$

which can be approximated by $I^{* h}$. Hence, the conclusion is analogous,

$$
\sum_{e=1}^{N}\left(S^{*}, \phi\right)_{\mathscr{K}_{e}}=\sum_{e=1}^{N}\left(S^{* h}, \phi\right)_{\mathscr{K}_{e}}-\left\langle\llbracket I^{* h} \rrbracket \boldsymbol{v} \cdot \boldsymbol{n}, \phi\right\rangle_{\partial \mathscr{K}_{e}-},
$$

where $S^{* h}:=\nabla I^{* h} \cdot \boldsymbol{v}+r^{-n}\left(I^{* h}-J\right) \in \mathscr{X}_{0}^{h}(\Omega)$, thus (4.6) turns into

$$
\begin{aligned}
\sum_{e=1}^{N}\left(\nabla \delta I^{h} \cdot \boldsymbol{v}, \phi\right)_{\mathscr{K}_{e}}+\left(r^{-n} \delta I^{h}, \phi\right)_{\mathscr{K}_{e}}-\left\langle\llbracket \delta I^{h} \rrbracket \boldsymbol{v} \cdot \boldsymbol{n}, \phi\right\rangle_{\partial \mathscr{K}_{e}^{-}} & = \\
- & \sum_{e=1}^{N}\left(\left(S^{h^{*}}, \phi\right)_{\mathscr{K}_{e}}-\left\langle\llbracket I^{* h} \rrbracket \boldsymbol{v} \cdot \boldsymbol{n}, \phi\right\rangle_{\partial \mathscr{K}_{e}-}\right) \quad \forall \quad \phi \in \mathscr{X}_{0}^{h}(\Omega) .
\end{aligned}
$$

Standard choices of a finite element basis for $\mathscr{X}_{0}^{h}(\Omega)$ are readily available CIARLET, 1978). The most attractive are discontinuous basis with compact support on the elements. Since trial, $\delta I^{h}$, and test, $\phi$, functions can be expressed as a linear combination of this basis, the final discontinuous Galerkin finite element approximation to the solution of (4.1) reads: find $\left.\delta I^{h}\right|_{\mathscr{K}_{e}} \in \mathscr{P}_{k}\left(\mathscr{K}_{e}\right)$ such that

$$
\begin{aligned}
& \left(\nabla \delta I^{h} \cdot \boldsymbol{v}, \phi\right)_{\mathscr{K}_{e}}+\left(r^{-n} \delta I^{h}, \phi\right)_{\mathscr{K}_{e}}-\left\langle\delta I_{+}^{h}, \boldsymbol{v} \cdot \boldsymbol{n}, \phi\right\rangle_{\partial \mathscr{K}_{e}^{-}}= \\
& -\left(\left(S^{h^{*}}, \phi\right)_{\mathscr{K}_{e}}-\left\langle I_{+}^{* h} \boldsymbol{v} \cdot \boldsymbol{n}, \phi\right\rangle_{\partial \mathscr{K}_{e}^{-}}+\sum_{e=1}^{N}\left\langle I_{-}^{h} \boldsymbol{v} \cdot \boldsymbol{n}, \phi\right\rangle_{\partial \mathscr{K}_{e}^{-}}\right) \quad \forall \quad \phi \in \mathscr{P}_{k}\left(\mathscr{K}_{e}\right)
\end{aligned}
$$


for all $\mathscr{K}_{e} \in \mathscr{T}^{h}$, where $I_{-}^{h} \equiv I_{-}^{* h}+\delta I_{-}^{h}$ was used. Note that this is almost an elementby-element algebraic set of linear equations for the unknown $\left.\delta I^{h}\right|_{\mathscr{K}}$. The only term preventing the simultaneous solution of (4.10) for each element is the sum (on the right side) for the value of $I_{-}^{h}$ in the neighbor elements of $\mathscr{K}_{e}$ sharing $\partial \mathscr{K}_{e}^{-}$. In some cases there exists a fast solution to this problem; this topic is covered next.

\subsection{Computational wave fronts and elemental linear systems}

The validity of (4.10) holds for general $\boldsymbol{v}$ fields and general partitions $\mathscr{T}^{h}$. However a computationally efficient implementation can be made if $\boldsymbol{v}$ is a "flow" without "recirculation", and the edges of $\mathscr{K}_{e}$ are either inflow or outflow of information (both inflow and outflow through the same edge are not allowed). From the characteristic curves in figure 4.1 it can be observed that no recirculation is present in the tranfer problem (2.1), and that a structured quadrilateral partition of $\Omega$ satisfies the condition of edge "uniflow."

Let the elements of $\mathscr{T}^{h}$ be ordered such that the element coupling term (sum) on the right side of (4.10) is known. That is all elements $\mathscr{K}_{d}$ with $d<e$ that share $\partial \mathscr{K}_{e}^{-}$ have been visited and solved for $I_{-}^{h}\left(=I_{-}^{* h}+\delta I_{-}^{h}\right)$. This ordering is attainable and must start at the inflow boundary with elements possessing $\partial \mathscr{K}_{e}^{-}$on $\partial \Omega^{-}$, therefore for this subset of boundary elements, $\mathscr{T}_{1}^{h}:=\left\{\mathscr{K}_{e} \mid e=N_{0}, \ldots, N_{1}\right\}$ (note that $\left.\phi\right|_{\partial \Omega^{-}} \equiv 0$ by construction), 4.10 reduces to

$$
\begin{aligned}
\left(\nabla \delta I^{h} \cdot \boldsymbol{v}, \phi\right)_{\mathscr{K}_{e}}+\left(r^{-n} \delta I^{h}, \phi\right)_{\mathscr{K}_{e}}-\left\langle\delta I_{+}^{h}, \boldsymbol{v} \cdot \boldsymbol{n}, \phi\right\rangle_{\partial \mathscr{K}_{e}^{-}}= & \\
-\left(S^{h^{*}}, \phi\right)_{\mathscr{K}_{e}} & \forall \quad \phi \in \mathscr{P}_{k}\left(\mathscr{K}_{e}\right),
\end{aligned}
$$

which can be solved for $\left.\delta I^{h}\right|_{\mathscr{K}_{e}}$ in every $\mathscr{K}_{e} \in \mathscr{T}_{1}^{h}$ simultaneously. Because the union of $\partial \mathscr{K}_{e}^{+}$for all elements in $\mathscr{T}_{1}^{h}$ form a new inflow boundary front, another group of elements whose inflow boundary lie on $\bigcup_{e} \partial \mathscr{K}_{e} \forall \mathscr{K}_{e} \in \mathscr{T}_{1}^{h}$ can be colored, say $\mathscr{T}_{2}^{h}:=$ $\left\{\mathscr{K}_{e} \mid e=N_{1}+1, \ldots, N_{2}\right\}$. The process can continue recursively until $\mathscr{T}^{h}=\bigcup_{i} \mathscr{T}_{i}^{h}$ with $\bigcap_{i} \mathscr{T}_{i}^{h}=\emptyset$. Each subset $\mathscr{T}_{i}^{h}$ holds a group of elements lying on a wave front so that the final ordering is indicated in figures 4.1 and 4.2 where the maximum number of element neighbors contributing to the sum in (4.10) is three. Despite the curved characteristic lines in the problem, a suitable sequencing of elements can be obtained by a modification of the ordering algorithm of LASAINT AND RAVIART (1974) (for straight characteristic curves). As the finite element partition is refined, particularly in the angular direction, the cardinality of $\mathscr{T}_{i}^{h}$ increases and many elements can be solved for simultaneously with exploitation of computational parallelism (fig. 4.2).

With the choice of a basis $\left\{w_{j} \mid j=1 \ldots, M_{e}\right\}$ for $\mathscr{P}_{k}\left(\mathscr{K}_{e}\right)$, trial and test functions become a linear combination of $w_{j}$ 's defined by unknown coefficients $x_{j}$, say $\left.\delta I^{h}\right|_{\mathscr{K}_{e}} \equiv$ 

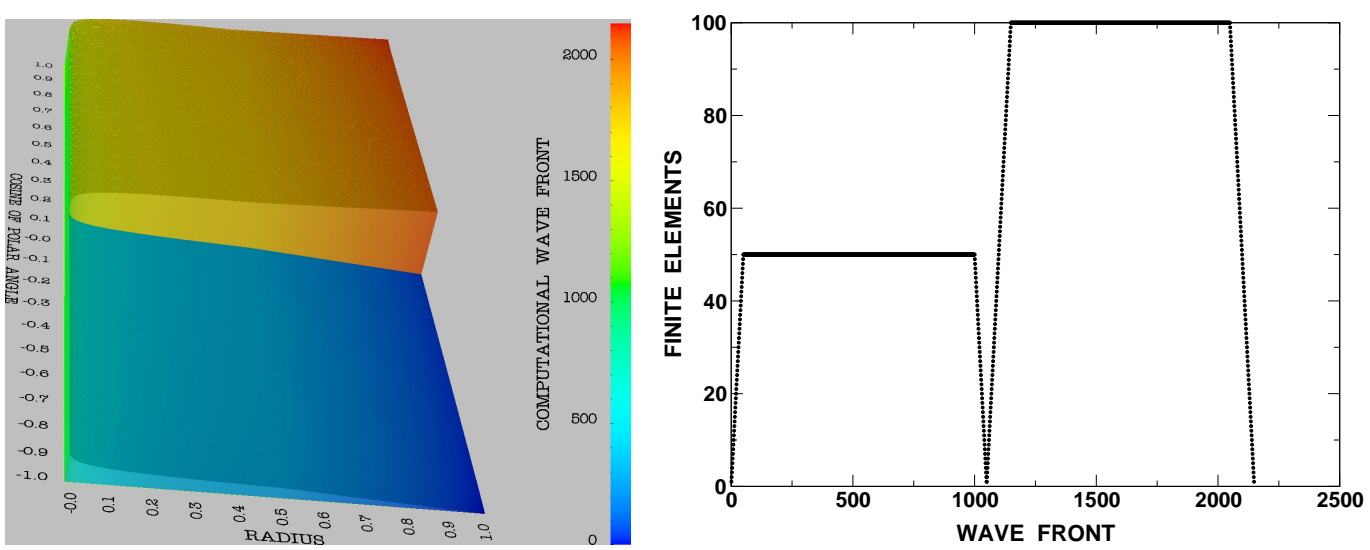

Fig. 4.2: Discontinuous Galerkin computational wave fronts. Mesh size is of 150000 structured quadrilateral elements. On the left, a particular color indicates a group of elements that can be solved simultaneously provided that all elements in the previous color level have been visited. There exits 2148 fronts (groups) of elements $\left(\mathscr{T}^{h}=\right.$ $\left.\bigcup_{i=1}^{2148} \mathscr{T}_{i}^{h}\right)$. On the right, a graph of the number of elements $N_{i}-N_{i-1}$ per front $\mathscr{T}_{i}^{h}$ is reported. In average, the lower part of the phase space (incoming radiation) can be solved for at a rate of 50 elements per wave front simultaneously. The upper part has a larger number of elements, in the angular direction, and can be solved at an average rate of 100 elements per front simultaneously.

$\left.\sum_{j=1}^{M_{e}} x_{j} w_{j}\right|_{\mathscr{K}_{e}}$. Upon substitution in 4.10 the following linear system of algebraic equations is obtained for element $\mathscr{K}_{e}$

$$
\sum_{j=1}^{M_{e}}{ }^{e} A_{i, j}{ }^{e} x_{j}=-{ }^{e} b_{i} \quad i=1, \ldots, M_{e}
$$

where

$$
\begin{aligned}
{ }^{e} A_{i, j} & :=\left(\nabla w_{j} \cdot \boldsymbol{v}, w_{i}\right)_{\mathscr{K}_{e}}+\left(r^{-n} w_{j}, w_{i}\right)_{\mathscr{K}_{e}}-\left\langle w_{j} \boldsymbol{v} \cdot \boldsymbol{n}, w_{i}\right\rangle_{\partial \mathscr{K}_{e}^{-}}, \\
{ }^{e} b_{i} & :=\left(S^{h^{*}}, w_{i}\right)_{\mathscr{K}_{e}}-\left\langle I_{+}^{* h} \boldsymbol{v} \cdot \boldsymbol{n}, w_{i}\right\rangle_{\partial \mathscr{K}_{e}^{-}}+\sum_{e=1}^{N}\left\langle I_{-}^{h} \boldsymbol{v} \cdot \boldsymbol{n}, w_{i}\right\rangle_{\partial \mathscr{K}_{e}^{-}} .
\end{aligned}
$$

The elemental matrix of coefficients ${ }^{e} A_{i, j}$ is typically dense so is the elemental vector ${ }^{e} b_{i}$. The number of basis functions $M_{e}$ depends on the polynomial space $\mathscr{P}_{k}$; for a common quadrilateral finite element $M_{e}=(k+1)^{2}$ (bi-linear polynomials lead to 
$\left.M_{e}=4\right)$. In view of the discontinuity property of these polynomials, the degree of polynomial approximation can be variable with the finite element without changes in any of the equations so far presented. Therefore this solution method, for the radiative transfer equation (3.1), lends itself easily to hp-adaptivity. Since the size of (4.12) is modest even for the largest practical polynomial degree imagined, a factorization method for computing $x_{j}$ is the best option.

The linear forms in (4.13) and (4.14) are computed by Gauss quadrature. If linear polynomials are used $\left(\mathscr{P}_{1}\right)$, the integrands become polynomials with degree less than or equal to 2, therefore 2 Gauss points per direction in phase space, that is a total of 4 Gauss points per quadrilateral finite element, is of sufficient accuracy.

Finally the wave front solution of $(4.10)$ is summarized in the following algorithm which replaces step 8 in algorithm (3.1).

Algorithm 4.1. Computational wave front sweeping algorithm

1. Partition $\mathscr{T}^{h}=\bigcup_{i=1}^{N_{f}} \mathscr{T}_{i}^{h}$ (preprocessing step done only once)

2. $I^{* h}(r, \mu)$ and $J(r)$ are available (see step 8 in algorithm 3.1)

3. For $i=1, \ldots, N_{f}$ Do:

4. $\forall \mathscr{K}_{e} \in \mathscr{T}_{i}^{h}$ Do: (simultaneously for each $\mathscr{K}_{e}$ )

5. $\quad$ Form ${ }^{e} A_{i, j}$ and ${ }^{e} b_{i}$ in (4.13) and (4.14)

6. Solve 4.12 for ${ }^{e} x_{j}$ and construct $\left.\delta I^{h}\right|_{\mathscr{K}_{e}}$

7. Update element solution $\left.\left.I^{h}\right|_{\mathscr{K}_{e}} \equiv I^{* h}\right|_{\mathscr{K}_{e}}+\left.\delta I^{h}\right|_{\mathscr{K}_{e}}$

8. EndDo

9. EndDo

\subsection{Finite element computation of moments}

Careful evaluation of moments of the radiative specific intensity (energy density, energy flux, and pressure) in steps 12-13 of algorithm 3.1 is critical to obtaining accurate solutions. Also, on the practical side, naive implementations of the algorithm for computing moments can easily dominate the total computing time. The essential quantities to be computed are the $p$ th moments $m_{p}(r):=\int_{-1}^{+1} I\left(r, \mu^{\prime}\right)\left(\mu^{\prime}\right)^{p} d \mu^{\prime}$, for $p=0,1,2$. This radial function needs to be available for computing the vector ${ }^{e} b_{i}$ (4.14) at all Gauss points $\boldsymbol{g}_{e}^{i}$ of every element $\mathscr{K}_{e} \in \mathscr{T}^{h}$. The appropriate approach is to compute the moments using the available finite element structure. Given a finite element $\mathscr{K}_{e}$, and a Gauss point $\boldsymbol{g}_{e}^{i}$, the first step is to find a (linked) list of long-range moment-neighbors $\mathscr{N}_{e}^{i} \subset \mathscr{T}^{h}$ indicated by the elements traversed by the dashed line $r=\left(\boldsymbol{g}_{e}^{i}\right)_{r}$ in figure 4.3. The search process starts by inserting $\mathscr{K}_{e}$ into the list $\mathscr{N}_{e}^{i}$ and finding the intersections of the dashed line with the edges of $\mathscr{K}_{e}$. If there is a valid intersection, the neighbor elements sharing that edge are inserted in the list $\mathscr{N}_{e}^{i}$. By finding the intersections of the neighbors with the dashed line recursively, $\mathscr{N}_{e}^{i}$ is 
quickly filled. As a bonus, the limits of the intervals $\ell_{e}^{i}$ (fig. 4.3) are automatically found and used, in companion with $\mathscr{N}_{e}^{i}$, to compute the $p$ th moment

$$
m_{p}\left(r=\left(\boldsymbol{g}_{e}^{i}\right)_{r}\right):=\sum_{\mathscr{K}_{f} \in \mathscr{N}_{e}^{i}} \int_{\ell_{f}^{i}} I\left(\boldsymbol{g}_{e}^{i}\right)\left(\left(\boldsymbol{g}_{e}^{i}\right)_{\mu^{\prime}}\right)^{p} d \mu^{\prime} .
$$

The above value can be saved into yet another conveniently searchable data structure to avoid re-computing the moment $m_{p}(r)$ at a radial point coincident with the radial component of the Gauss point belonging to a previously visited finite element.

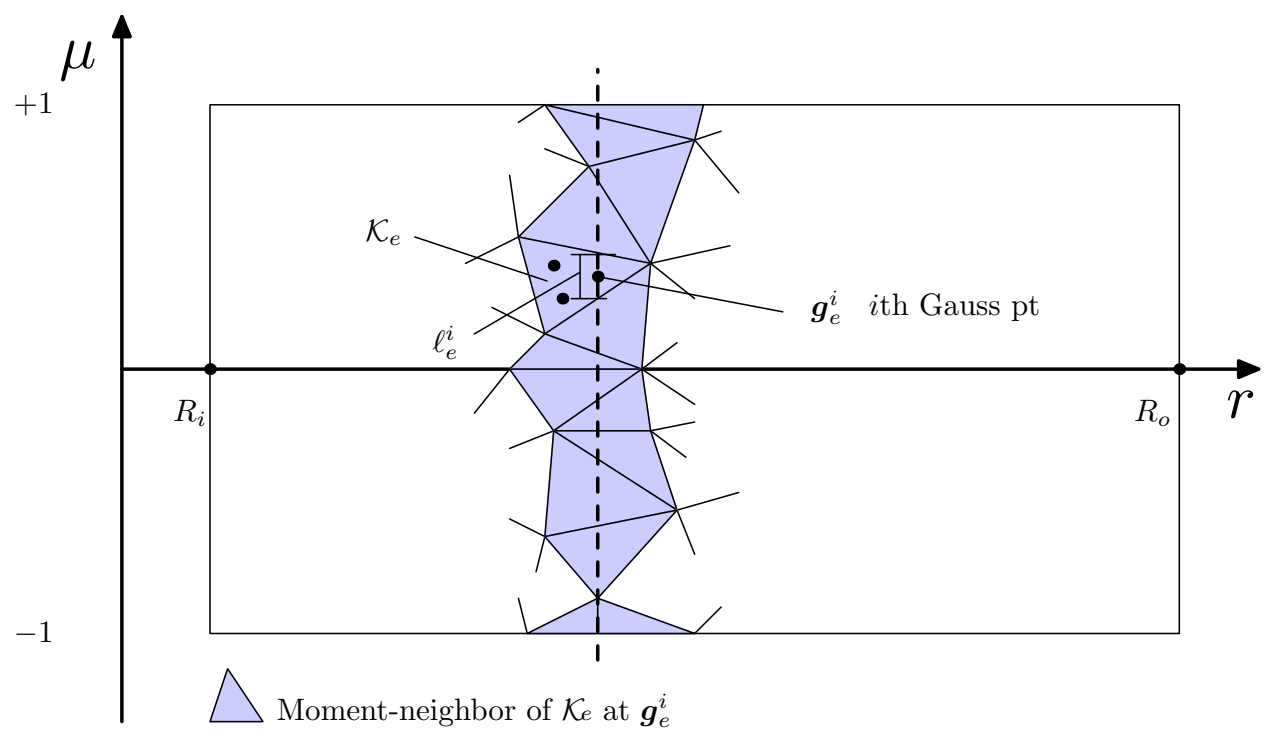

Fig. 4.3: Finite element computation of moments of the radiative intensity. The long-range moment-neighbors of an element at its ith Gauss point are all the elements intersected by the dashed line passing through that point.

\section{The radiative momentum problem}

The solution of the governing equation for radiative pressure (3.2) at the $k$ th iteration in algorithm (3.1) can also be constructed by the discontinuous Galerkin method (sec.4.1). For this one-dimensional problem, the method simplifies greatly (LASAINT AND RAVIART, 1974) and the element ordering and direction of sweeping is $R_{o} \rightarrow R_{i}$. A detail of practical importance is the definition of the finite element partition of the interval $\left[R_{o}, R_{i}\right]$ in configurational space. It is advantageous to inherit the partition from the collection of underlying Gauss points used in phase space (fig. 5.1), and 
to use piecewise constant basis $\mathscr{P}_{0}$ for the finite element approximation of $(3.2)$. Therefore one algebraic equation per element in the radial direction is obtained, and the solution is straightforward. As a result, step 15 (algorithm3.1), which computes the energy density field from the pressure field in preparation for the solution of the radiative transfer equation (3.1), turns into a simple matter of distributing the computed values directly into the elemental data required to compute the first term of ${ }^{e} b_{i}$ in 4.14 .

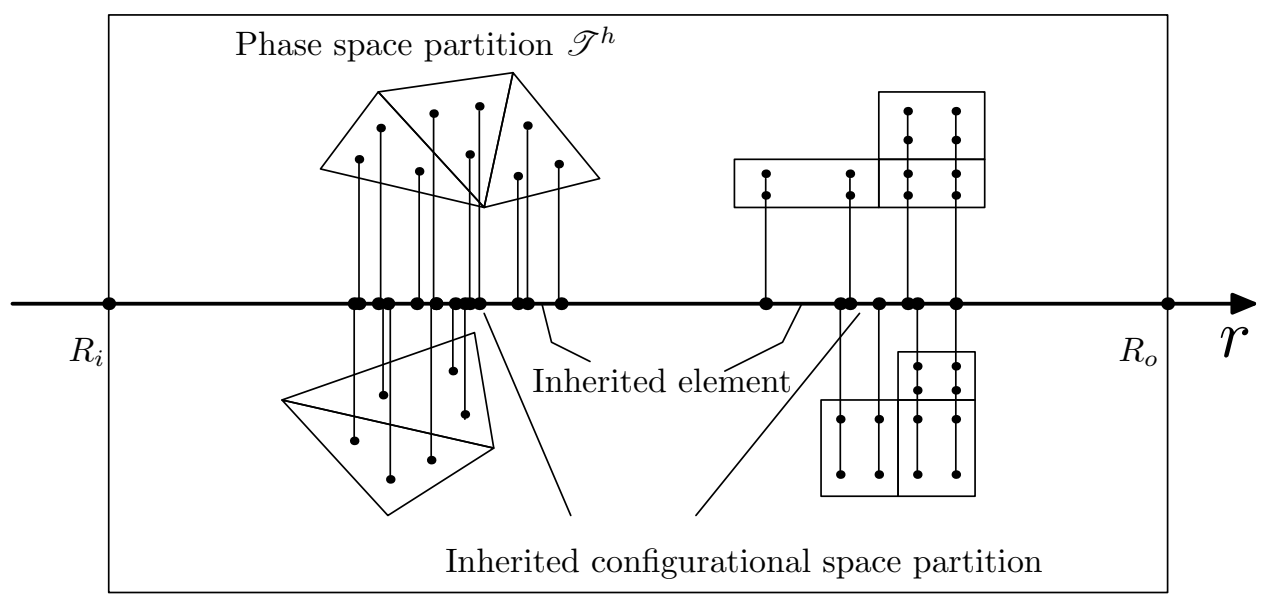

Fig. 5.1: Inherited-by-projection pressure mesh on the interval $\left[R_{o}, R_{i}\right]$. Pressure mesh element boundaries are defined by the $r$ coordinates of all Gauss points of elements in the phase space.

\section{Results and discussion}

Four sizes of stellar atmosphere were studied with power law scattering exponents $n=2,3,4,6,8$, and 10 . In all cases (table 6.1) the inner boundary was placed at $R_{i}=10^{-3}$, measured in units of $\alpha^{1 /(n-1)}$ (refer to scaling in sec. 2.3), where the optical depth was large enough $\left(\tau\left(R_{i}\right)>1000\right)$ to justify the diffusive inner boundary condition ( $\sec 2.2$ ). The length of the outer radius was varied three orders of magnitude (fig. 6.1) to examine diffusively dominated atmospheres (case 1) up to point-source extended atmospheres (case 4). The diffusive-transparent transition region is indicated by $r_{t}(2.22)$ values in parenthesis in table 6.1. Note that the position of the transition region has a minimum for the range of $n$ studied when $R_{o} \geq 1$. By virtue of the scaling adopted, the significance of a particular radial value $r$ is that the mean free path of a photon emitted at that position is $r^{n}$. 
Table 6.1: Order of magnitude of the optical depths of inner boundaries $\tau\left(R_{i}\right)$, and radius of transition region $\tau\left(r_{t}\right)=1$ (truncated value in parenthesis), for various scattering power law indices $n$. Radius measured in units of $\alpha^{1 /(n-1)}$. See section 2.3.5 for formulae.

\begin{tabular}{|c|c|c||c|c|c|c|c|c|}
\hline Case & $R_{i}$ & $R_{o}$ & $n=2$ & $n=3$ & $n=4$ & $n=6$ & $n=8$ & $n=10$ \\
\hline \hline 1 & $10^{-3}$ & 0.1 & $10^{3}(.09)$ & $10^{5}(.09)$ & $10^{8}(.09)$ & $10^{14}(.09)$ & $10^{20}(.09)$ & $10^{26}(.09)$ \\
2 & $10^{-3}$ & 1 & $10^{3}(.50)$ & $10^{5}(.57)$ & $10^{8}(.62)$ & $10^{14}(.69)$ & $10^{20}(.74)$ & $10^{26}(.77)$ \\
3 & $10^{-3}$ & 10 & $10^{3}(.90)$ & $10^{5}(.70)$ & $10^{8}(.69)$ & $10^{14}(.72)$ & $10^{20}(.75)$ & $10^{26}(.78)$ \\
4 & $10^{-3}$ & 100 & $10^{3}(.99)$ & $10^{5}(.70)$ & $10^{8}(.69)$ & $10^{14}(.72)$ & $10^{20}(.75)$ & $10^{26}(.78)$ \\
\hline
\end{tabular}

CPU time and memory requirement data presented in table $6.2(n=2)$, obtained through POSIX functions, indicate computational resources required in various computer platforms. No elaborated implementation/optimization of the algorithm was advanced beyond what is naturally induced by the solution method. The solution of the elemental linear systems 4.12 was computed with the LAPACK library routine dgesvx.

\subsection{Phase space finite element partition}

A bi-linear quadrilateral $\left(M_{e}=4\right)$ partition of $\Omega$ with 150000 elements $(600000$ algebraic equations) was created for all cases (fig. 6.2) with attention to clustering elements in regions of singularity and/or steep gradients. In the radial direction elements were clustered near the diffusive-transition region $\left(\tau\left(r_{t}\right)=1\right)$ where the radiative intensity decays exponentially, and near the inner boundary. In the angular direction, elements were accumulated in the region near $\theta=0$ for extended atmospheres (fig. 6.3) to capture the forward peaking of the radiative intensity. Elements were also clustered near $\theta=\pi$ for "cosmetic" reasons when creating a polar mapping of the phase space (figs. 6.5 and 6.6).

\subsection{Specific radiative intensity}

The radiative specific intensity is a highly exponentially decaying function of the radial position. Qualitative features of the solution can be promptly observed by mapping the phase space into a polar region where the interval $\left[R_{i}, R_{o}\right]$ is conveniently 


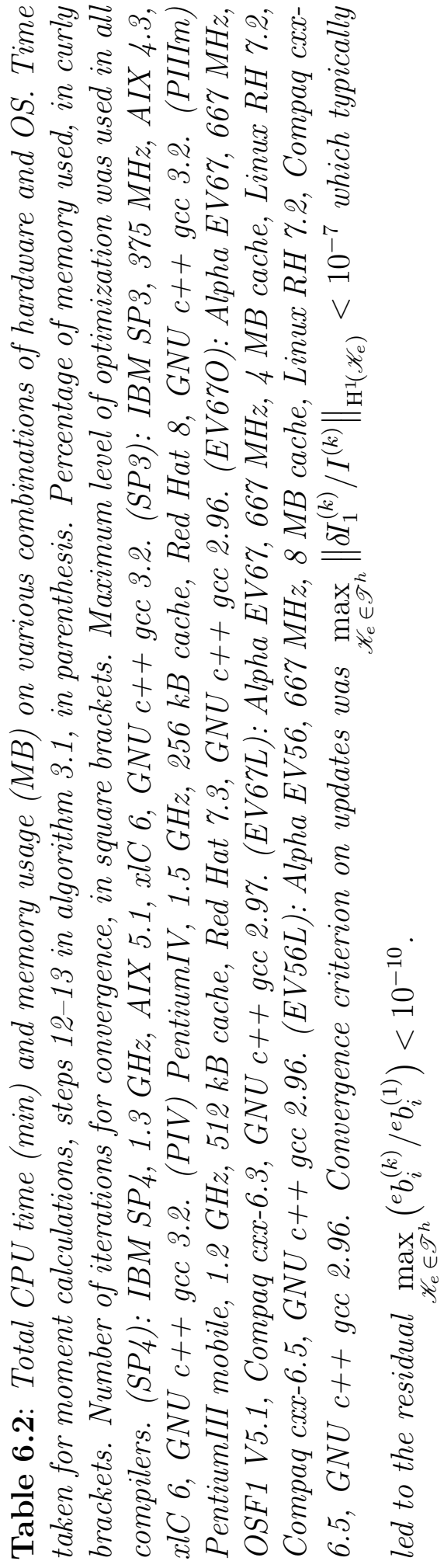

\begin{tabular}{|c|c|c|c|c|c|c|c|c|c|c|c|}
\hline $\begin{array}{l}+ \\
+ \\
\infty \\
\infty_{0} \\
\tilde{\rho}_{n}\end{array}$ & 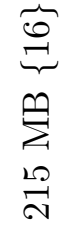 & $\begin{array}{l}\underset{g}{g} \\
\stackrel{g}{R}\end{array}$ & $\begin{array}{l}\underset{8}{\stackrel{2}{+}} \\
8\end{array}$ & 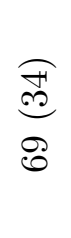 & $\underbrace{\infty}_{\kappa}$ & 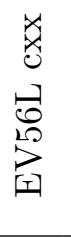 & 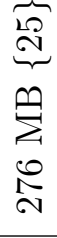 & 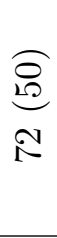 & 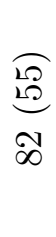 & 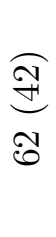 & 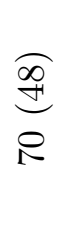 \\
\hline 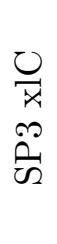 & $\begin{array}{l}\underbrace{N}_{\sim} \\
\underset{\Sigma}{\Sigma} \\
\stackrel{20}{\sim}\end{array}$ & 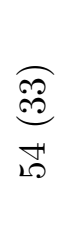 & $\begin{array}{l}\widehat{0} \\
\stackrel{8}{0} \\
0\end{array}$ & 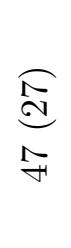 & $\underset{\text { in }}{\stackrel{\widehat{0}}{0}}$ & 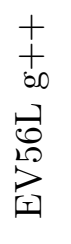 & 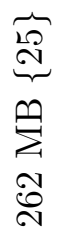 & 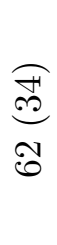 & $\begin{array}{l}\underset{\stackrel{\theta}{f}}{P} \\
\stackrel{P}{i}\end{array}$ & $\begin{array}{l}\stackrel{\widehat{\curvearrowright}}{0} \\
\overrightarrow{10}\end{array}$ & 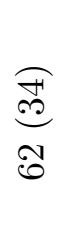 \\
\hline 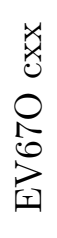 & 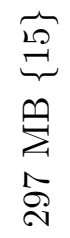 & 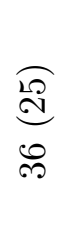 & 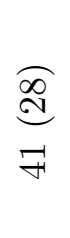 & $\underset{\mathscr{m}}{\stackrel{\overbrace =}{\mathscr{N}}}$ & 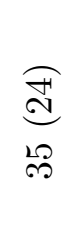 & 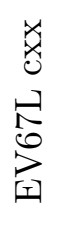 & 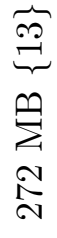 & 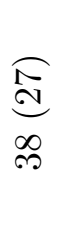 & 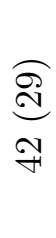 & 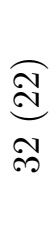 & 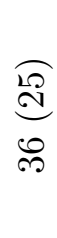 \\
\hline $\begin{array}{l}+ \\
+ \\
+\infty \\
\vec{\sigma} \\
\vec{\Omega}\end{array}$ & 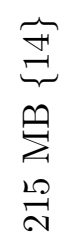 & 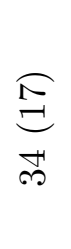 & 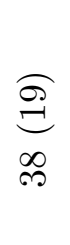 & $\begin{array}{l}\stackrel{\sqrt{2}}{\rightleftharpoons} \\
\stackrel{\infty}{\infty}\end{array}$ & $\underset{\infty}{\stackrel{0}{0}}$ & 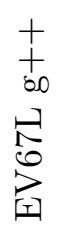 & 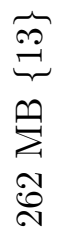 & 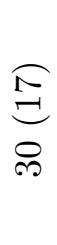 & 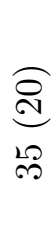 & 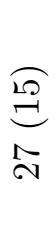 & 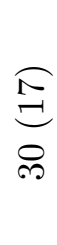 \\
\hline 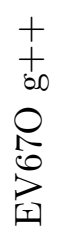 & 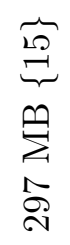 & $\begin{array}{l}\underset{\sigma}{\stackrel{\sigma}{g}} \\
\vec{\infty}\end{array}$ & 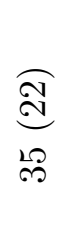 & $\underset{\stackrel{N}{N}}{\stackrel{ }{\approx}}$ & $\begin{array}{l}\underset{\sigma}{\Xi} \\
\stackrel{\rho}{\rho}\end{array}$ & $\begin{array}{l}+ \\
+ \\
\text { so } \\
\Xi \\
\exists \\
\exists\end{array}$ & $\underbrace{\overbrace{\substack{0 \\
\infty}}^{\infty}}_{\substack{\infty \\
\sum_{-1}^{\infty}}}$ & $\begin{array}{l}\underset{J}{\leftrightarrows} \\
\stackrel{\sim}{H}\end{array}$ & 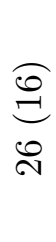 & $\begin{array}{l}\underset{\text { ㄱ }}{\beth} \\
\stackrel{\sim}{\sim}\end{array}$ & 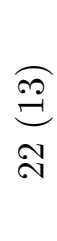 \\
\hline $\begin{array}{l}\frac{\partial}{x} \\
\vec{H} \\
\overrightarrow{\sigma_{n}}\end{array}$ & 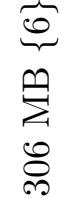 & 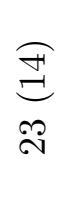 & 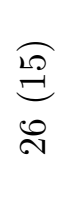 & $\begin{array}{l}\underset{\Im}{\Xi} \\
\vec{\sim}\end{array}$ & 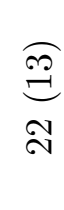 & $\begin{array}{l}+ \\
+ \\
\infty 0 \\
\overrightarrow{a_{1}}\end{array}$ & 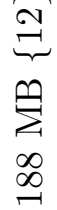 & 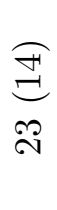 & $\begin{array}{l}\stackrel{10}{\rightleftharpoons} \\
\stackrel{0}{\sim}\end{array}$ & $\begin{array}{l}\underset{\Xi}{\Xi} \\
\text { 尺) }\end{array}$ & 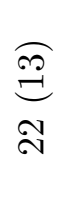 \\
\hline 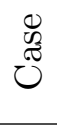 & $\$$ & $\underset{\Xi}{\Xi}$ & $\underset{\sim}{\stackrel{\infty}{\oplus}}$ & $\underset{\infty}{\stackrel{\sigma}{\sigma}}$ & $\underset{\forall}{\Xi}$ & ర్ల & $\$$ & $\underset{\exists}{\beth}$ & $\underset{N}{\stackrel{\infty}{=}}$ & $\frac{\sigma}{\underset{\sigma}{\ominus}}$ & $\underset{F}{\Xi}$ \\
\hline
\end{tabular}




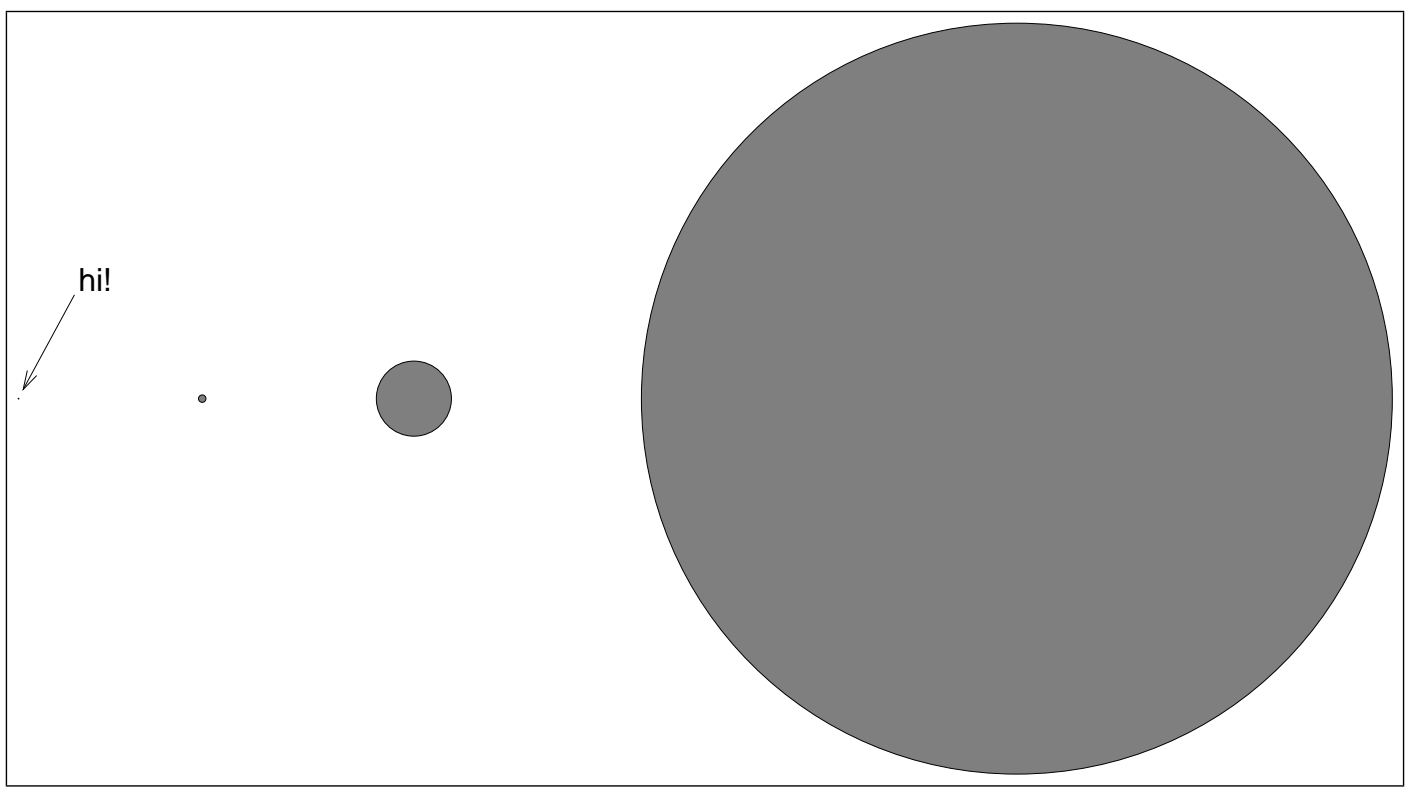

Fig. 6.1: Stellar atmosphere sizes analyzed. Far left, $R_{o}=0.1$; far right, $R_{o}=100$; in between (from left to right), $R_{o}=1$, and $R_{o}=10$. In table 6.1, $r_{t}$ indicates the radius of the diffusive-transparent transition region.

stretched via a logarithm-based function (fig. 6.4). Additional visual insight is obtained by wrapping the phase space around using the reflected mirror image in the lower half of the polar region. This mapping is particularly effective if the radiative intensity $I(r, \mu)$ is normalized by its value in the forward radial direction, that is $I(r, 1)$. This is an extension of the normalization used to evaluate the law of darkening of the emergent radiation (limb darkening curves) $I\left(R_{o}, \mu\right) / I\left(R_{o}, 1\right)$ CHANDRASEKHAR, 1960) to the entire radiation field; the end effect is shown in figure 6.5. The radiative intensity is shown as a continuous function by performing a simple average of the computed discontinuous values at the corners of the elements. In those images red is the maximum, one, and blue is the minimum, zero. Colors at points with different radii cannot be compared since they are normalized by different values. Therefore one should fix a particular radius and vary the direction $\theta$ to make quantitative comparisons. When looking at the normalized radiative intensity in the log-polar domain (fig. 6.5) one should keep in mind the "unstretched" configuration of figure 6.1.

The red colored region in the fairly flat top of the specific intensity surface (fig. 6.5) within $r_{t}$ represents a diffusive dominated region. When the atmosphere is small and essentially all diffusive (fig. 6.5 a), the flat top accounts for most of the surface except very near the outer boundary where non-isotropy induced by the boundary condition 


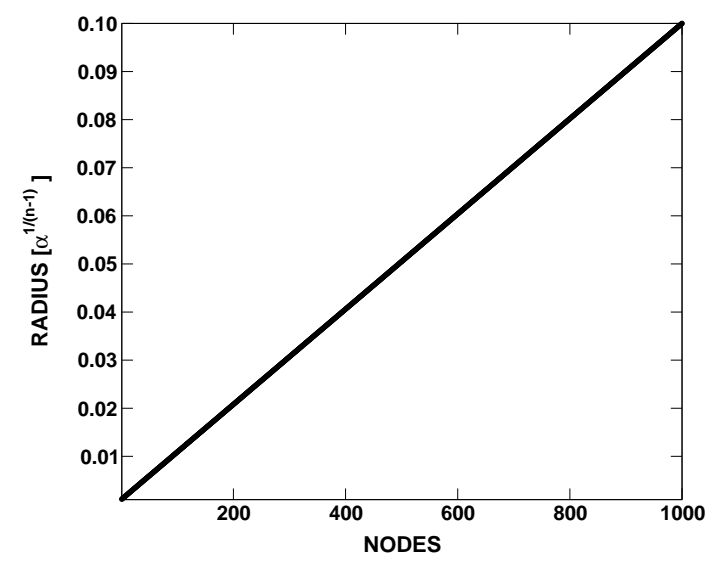

(a)

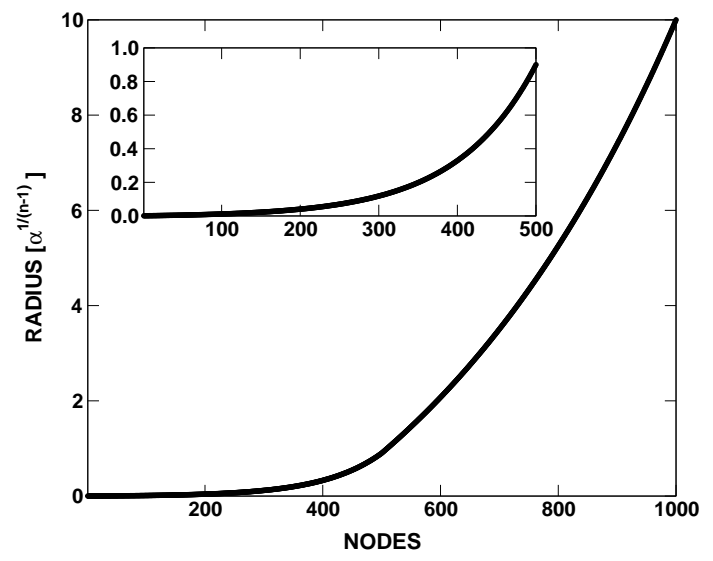

(c)

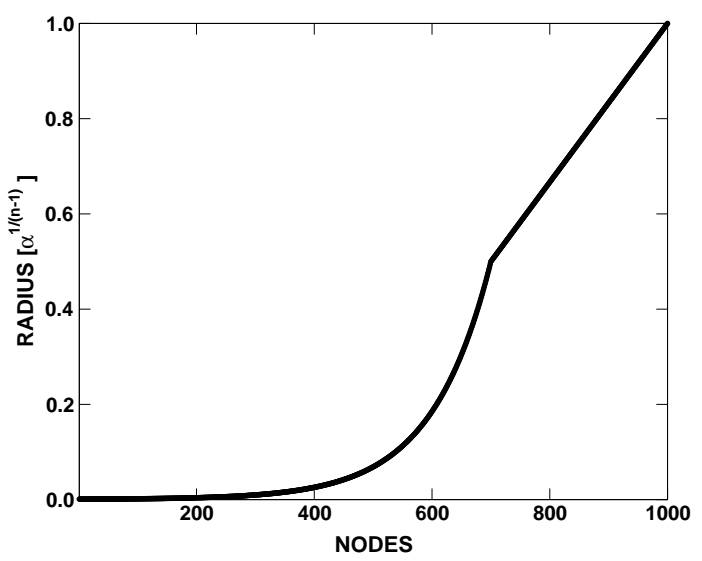

(b)

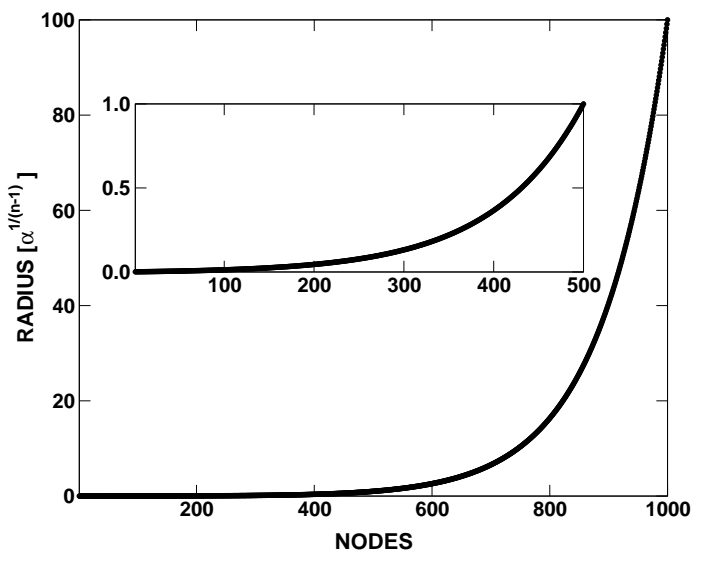

(d)

Fig. 6.2: Radial mesh distribution of finite elements for all study cases. (a) Linear distribution of 1000 elements along $r$ direction for case $R_{o}=0.1$. (b) Case $R_{o}=1$ with non-linear distribution of 700 elements within the diffusive region $(r \leq 0.5)$; the remaining 300 elements linear distributed. (c) Diffusive region ( $r \leq 0.9$ ) in case $R_{o}=10$ meshed with 500 elements non-linearly distributed. (d) Case $R_{o}=100$ with diffusive region $(r \leq 0.9)$ meshed with 500 elements non-linear distributed. 


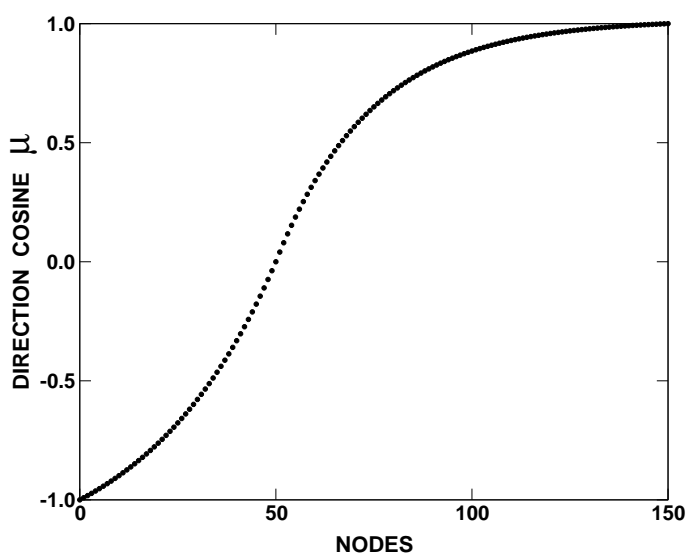

(a)

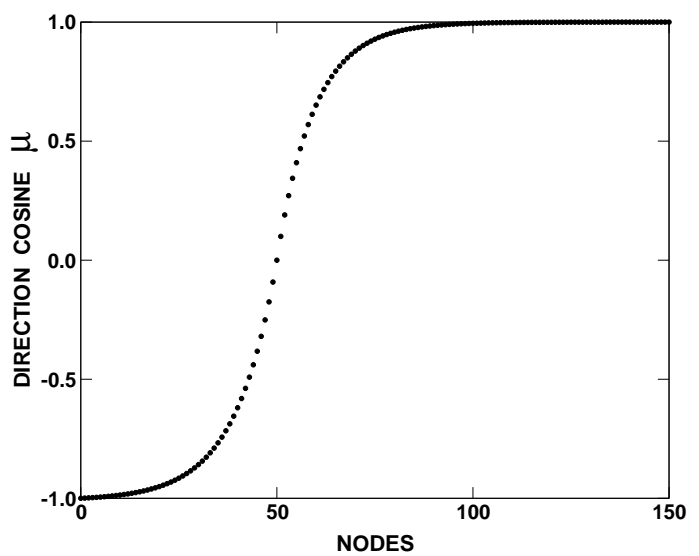

(c)

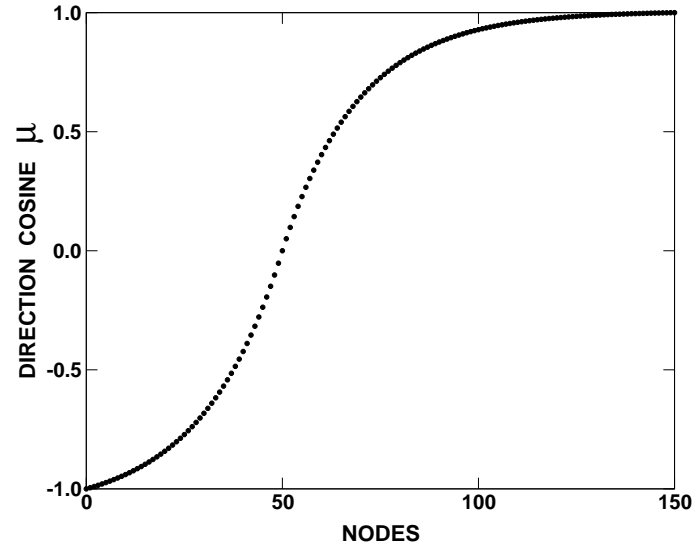

(b)

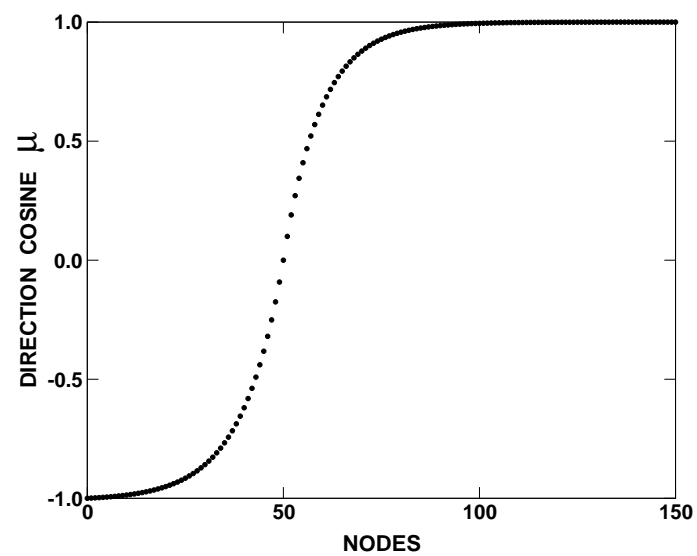

(d)

Fig. 6.3: Angular mesh distribution of finite elements for all study cases. The lower half of the phase space was partitioned with 50 elements, while the upper half, with 100. Elements are clustered towards $\mu=1$ in extended atmospheres to resolve the forward peaking behavior of the radiation intensity. (a) $R_{o}=0.1$. (b) $R_{o}=1$. (c) $R_{o}=10$. (d) $R_{o}=100$. 


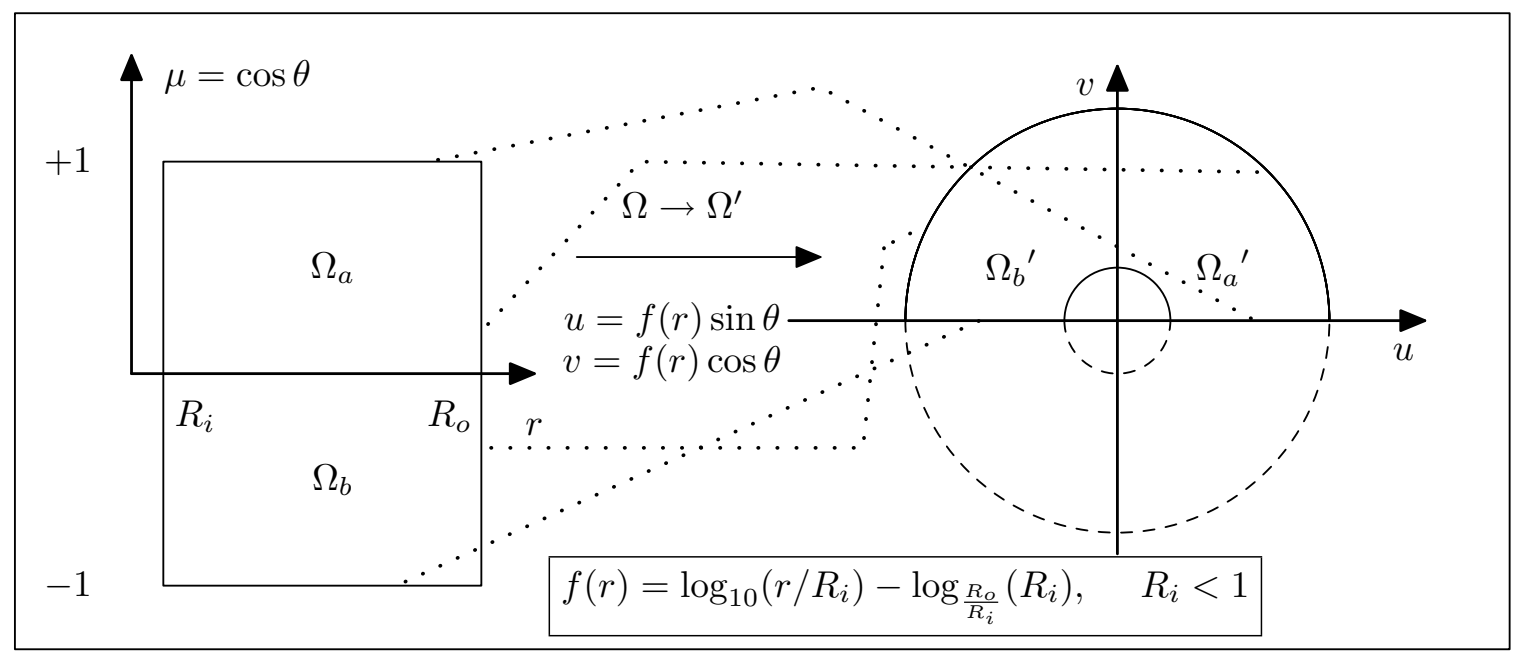

Fig. 6.4: Log-polar transformation of phase space provides visual insight into the radiative specific intensity (refer to fig. 6.5 and text).

takes place. As the atmosphere grows, non-isotropy is induced by the increasing transparency (inverse power law scattering) of the medium. For instance for $R_{o}=1$ non-isotropy is observable (fig. 6.5b) near the outer boundary (red to blue region). In larger atmospheres, $R_{o}=10$ (fig. 6.5c), limb darkening (flat blue ring) and diffusivetransparent transition (red to green steep gradient) is noticeable. The onset of forward peaking is clear as indicated by the forming delta function (sec. 2.1.5, eq. 2.10) at $\theta=0$ and $r \approx R_{o}$. When the atmosphere is further extended (fig. 6.5d) these features are enhanced; notably the forward peaking. Note however that the forward peaking is a qualitative feature of the solution which would not be easily observable without normalization since the magnitude of the peak vanishes with increasing radius $(2.20)$.

Increasing the scattering exponent, a diffusive region becomes more diffusive, hence the radiative intensity surface becomes flater. Concurrently, a transparent region turns into more transparent, therefore the gradient of the radiative intensity at the corresponding transition region becomes steeper (fig. 6.6).

A closer look at the results for limb darkening and forward peaking (fig. 6.7) shows how critical is the distribution of mesh elements near $\theta \approx 0$ in extended atmospheres in order to capture the forming delta-function behavior (fig. 6.7c-d). The graphs show the portion of emitted radiation streaming from optical depths less than one as a function of angular direction. 


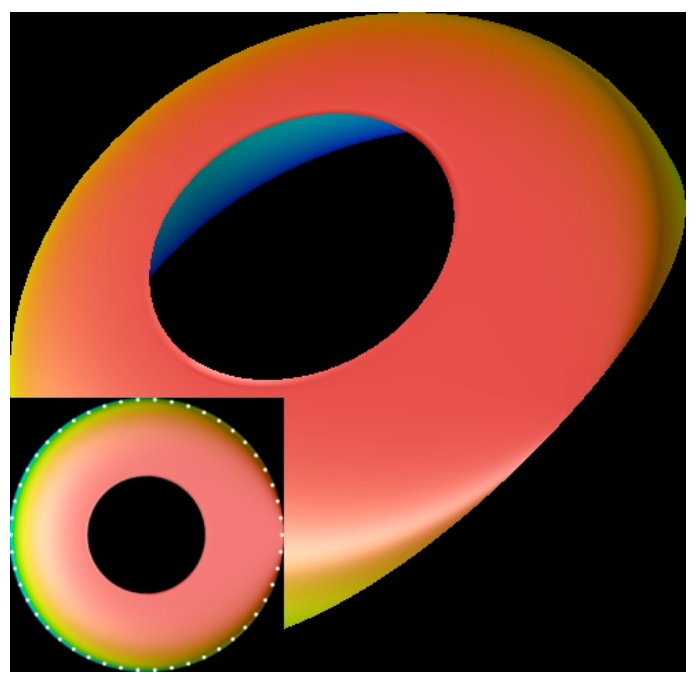

(a)

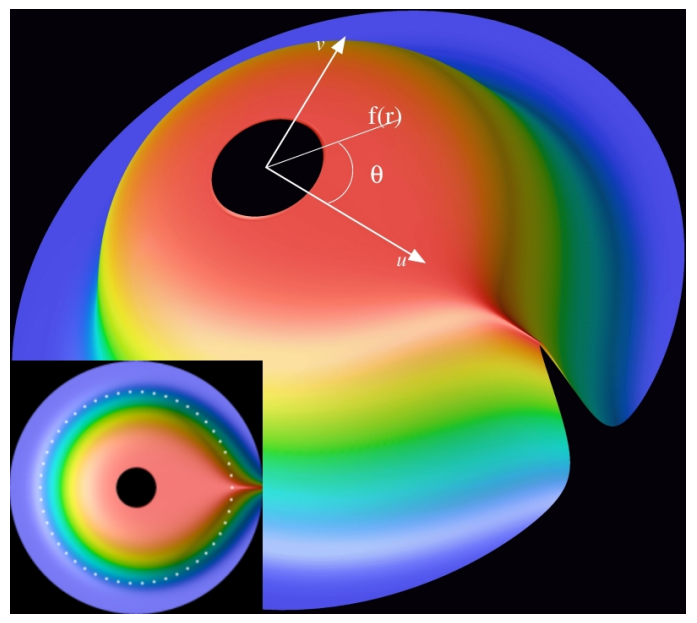

(c)

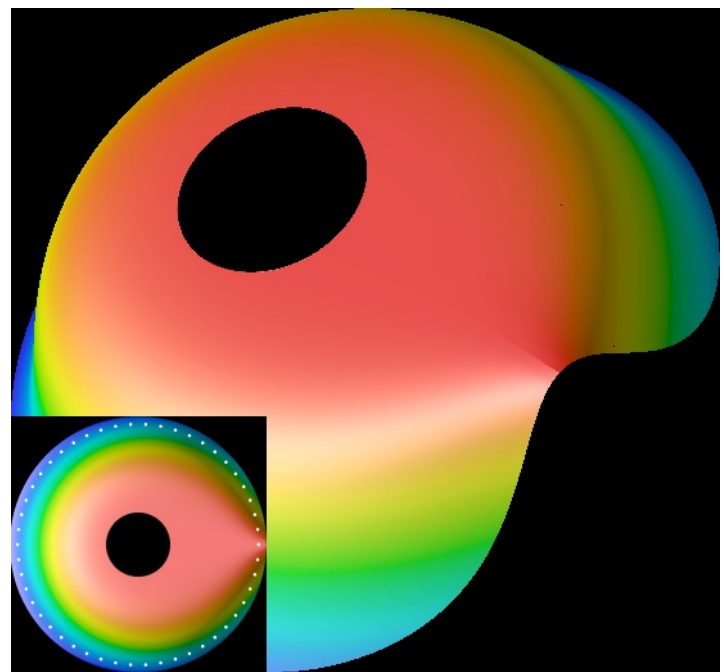

(b)

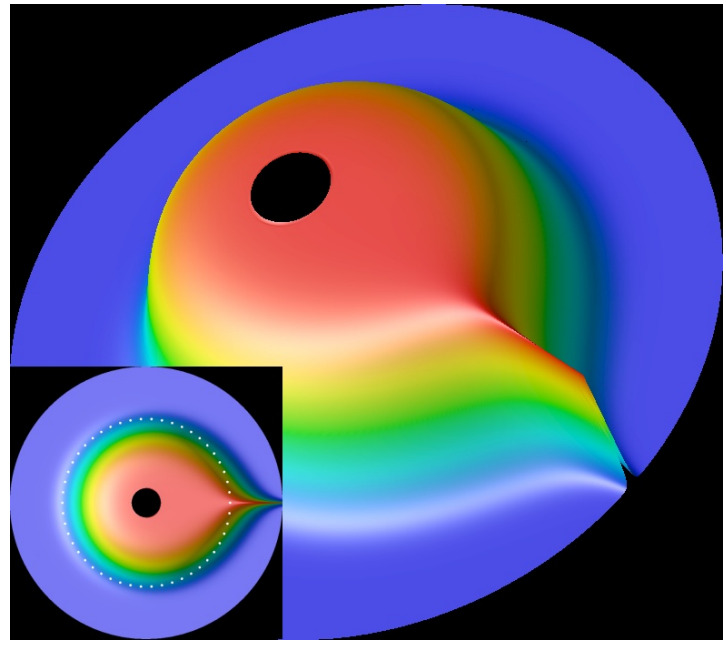

(d)

Fig. 6.5: Specific intensity normalized by the local intensity in the forward radial direction $(n=2)$. Lower left shows onset of forward peaking $(\theta=0)$. Lower right shows well developed forward peaking. Blue region in lower row indicates limb darkening. Top row shows diffusive-dominated radiation (red) except at the outer boundary $\left(r_{t}\right.$ indicated by dotted circle) where the non-isotropic effect from the boundary condition takes place. Center holes indicate the removal of the singularity at the origin. The region of steep gradient (light green) lies in the transition radius $r_{t}$. (a) $R_{o}=0.1$. (b) $R_{o}=1$. (c) $R_{o}=10$. (d) $R_{o}=100$. 

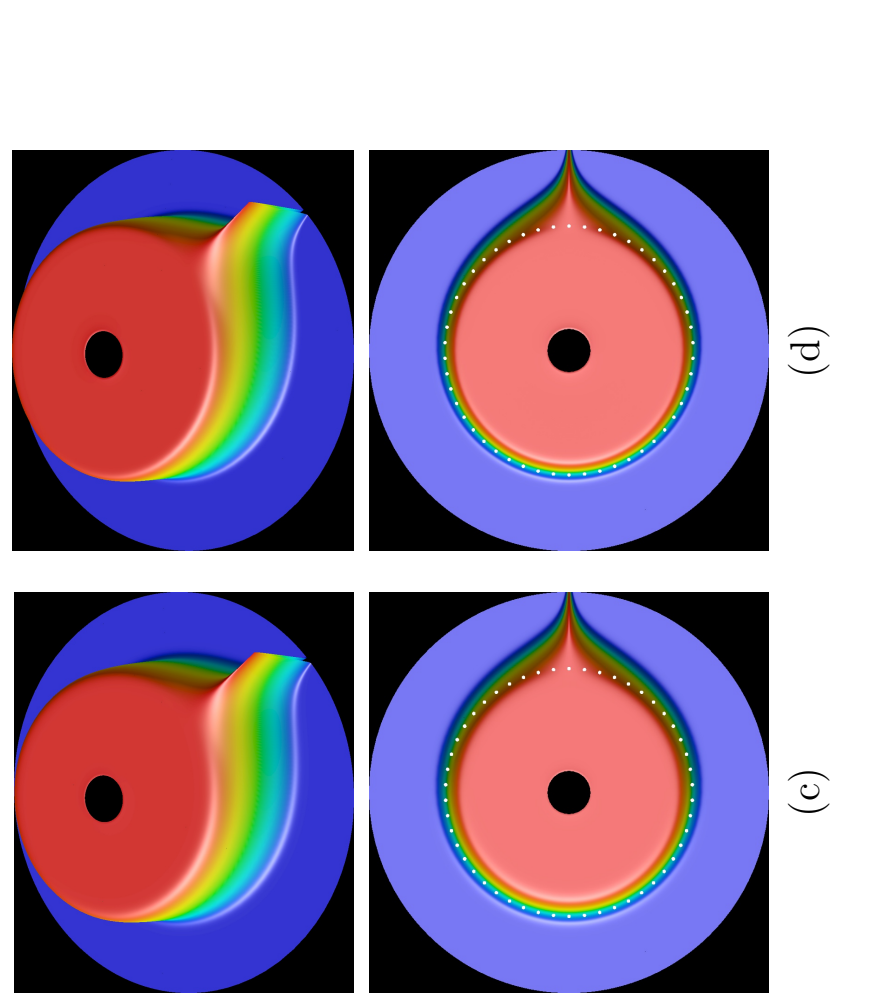

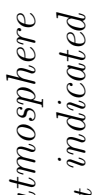

ग)

ญ్ है

ริ

. ह छ

$\therefore+\frac{1}{2}$

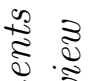

กิ

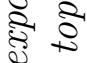

उ

e

离

ปั

के ई

क จ
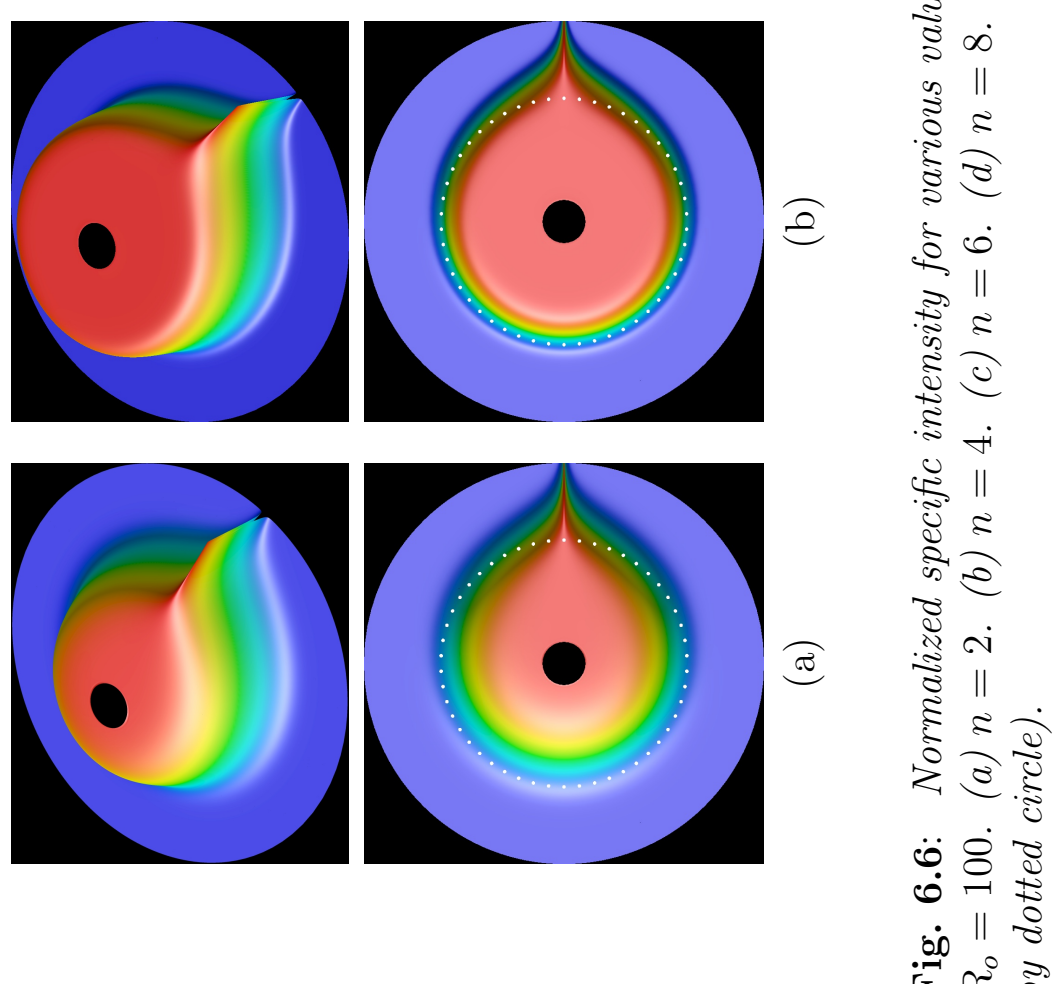

Oak Ridge National Laboratory Technical Report ORNL/TM-2003/072 pp. $1-44$ 


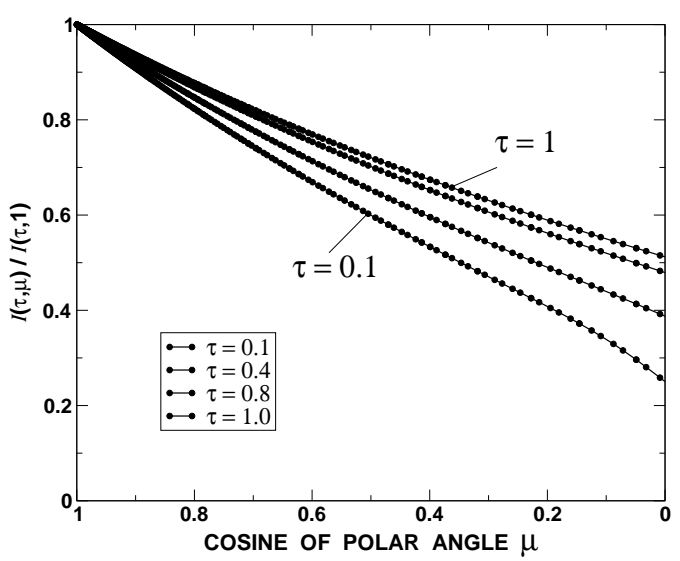

(a)

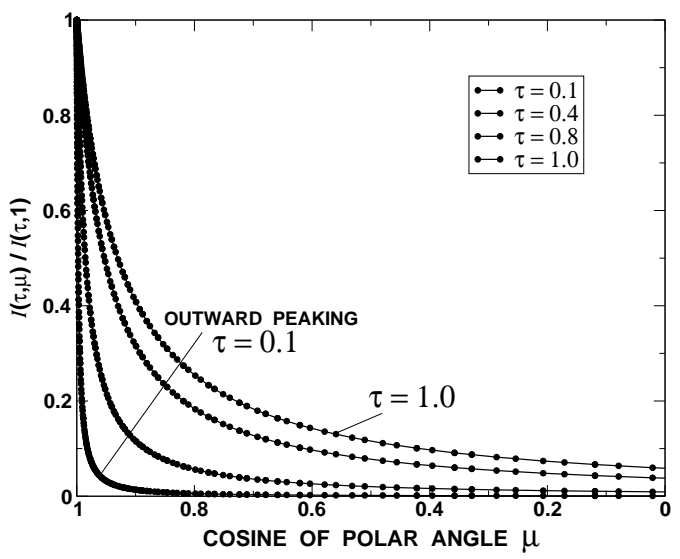

(c)

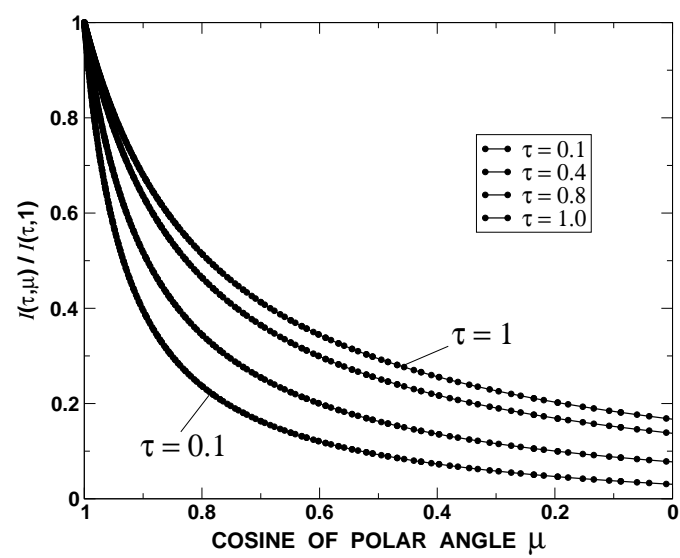

(b)

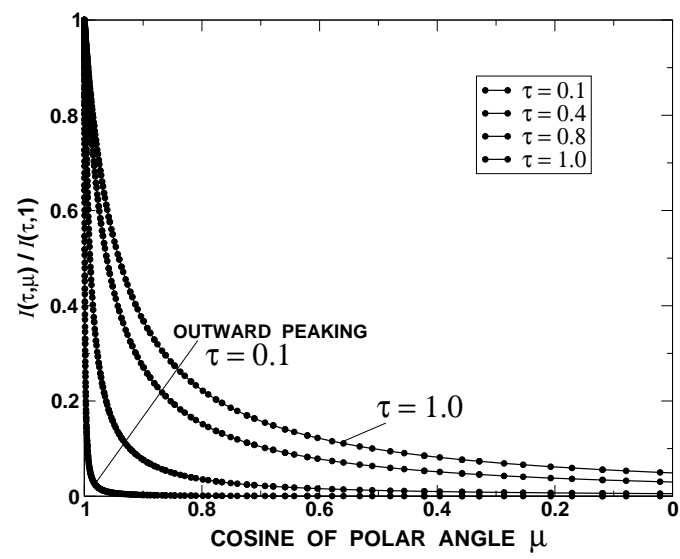

(d)

Fig. 6.7: Limb darkening and law of darkening for $n=2$. Graphs show normalized radiative intensity in the free streaming region $(\tau<1)$. Points on the curves indicate the projection of Gauss points from the phase space onto the $\mu$ half-axis. Note that $\tau=0.1$ is "near" the outer boundary and not on the outer boundary; at $\tau=10^{-4}$ forward peaking is even more pronounced (not shown). (a) $R_{o}=0.1$. (b) $R_{o}=1$. (c) $R_{o}=10$. (d) $R_{o}=100$. 


\subsection{Energy and derived quantities}

Energy density, energy flux (luminosity), and stress (pressure) are key quantities derived from the specific radiative intensity; together they couple radiative transfer to hydrodynamics and set the foundation of continuum stellar flow modeling. Here asymptotic analytical results (sec. 2.1) are correctly obtained and deviations from those results in the non-asymptotic limit are shown (all results are scaled as in sec. 2.3).

Energy density and pressure are decreasing functions of radius (fig. 6.8), unlike the undiluted energy flux (luminosity) which is a constant (fig. 6.9). The results shown for luminosity are calculated from the computed specific radiative intensity. Small $(\leq 10 \%)$ oscillations are observed (fig. 6.9) at optical depths greater than 100 . It is conjectured that the oscillations result from truncation errors obtained when subtracting large numbers during the calculation of the first (odd) moment of the radiative intensity at large optical depths (no oscillations are present in zeroth and second moments).

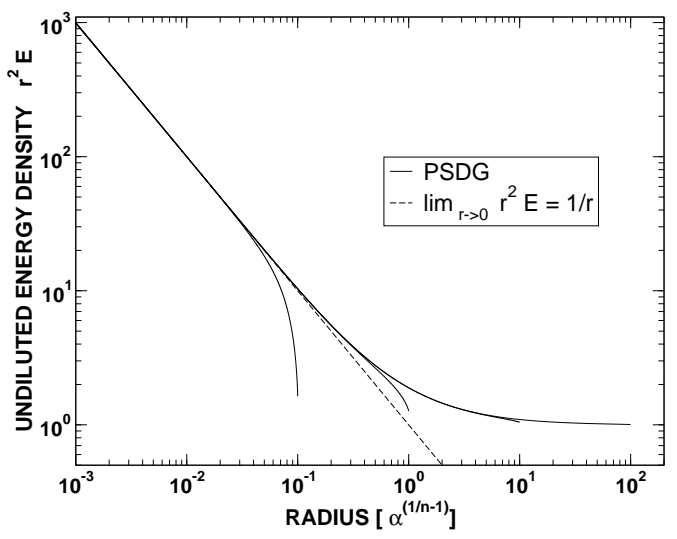

(a)

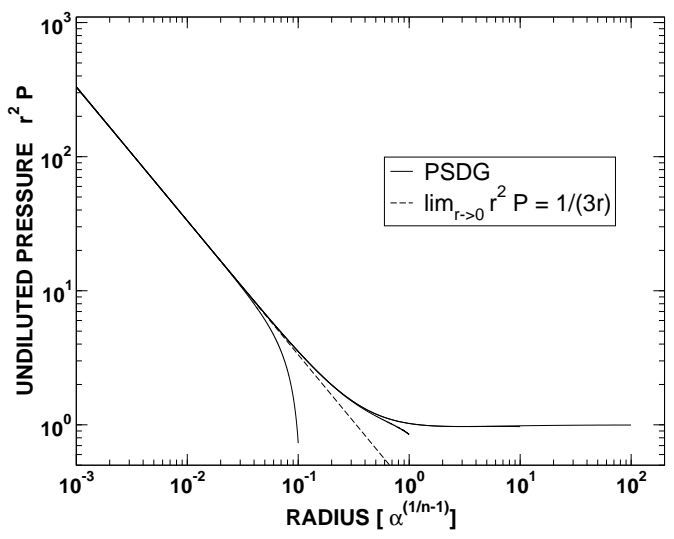

(b)

Fig. 6.8: Undiluted energy density and pressure $(n=2)$; measured in units of $L_{0} /(4 \pi c)$. Dashed line is the asymptotic diffusive limit at small radii. For extended atmospheres the streaming limit value for large radii is one (sec. 2.3.3). The PSDG method captures both limits accurately. The terminal point in each curve indicates the atmosphere size. (a) Energy density. (b) Pressure.

The calculated state of stress as represented by the invariants of the stress tensor (fig. 6.10) show the expected asymptotic behavior (2.9), namely, isotropic at large optical depth, and singular at the outer boundary for extended atmospheres; note 


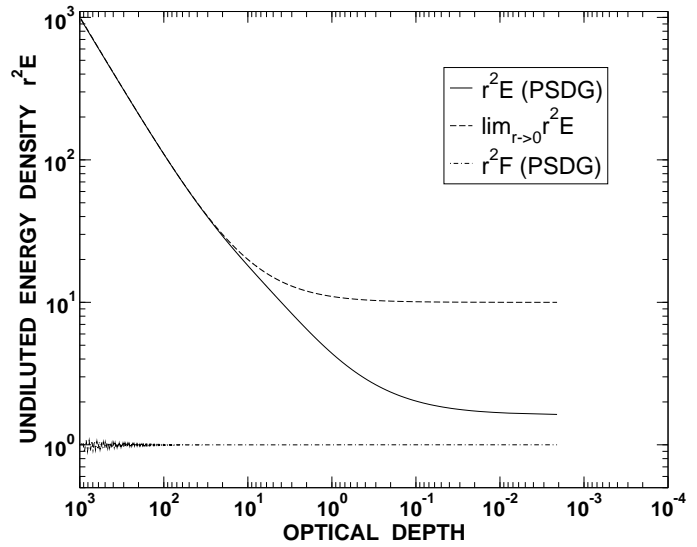

(a)

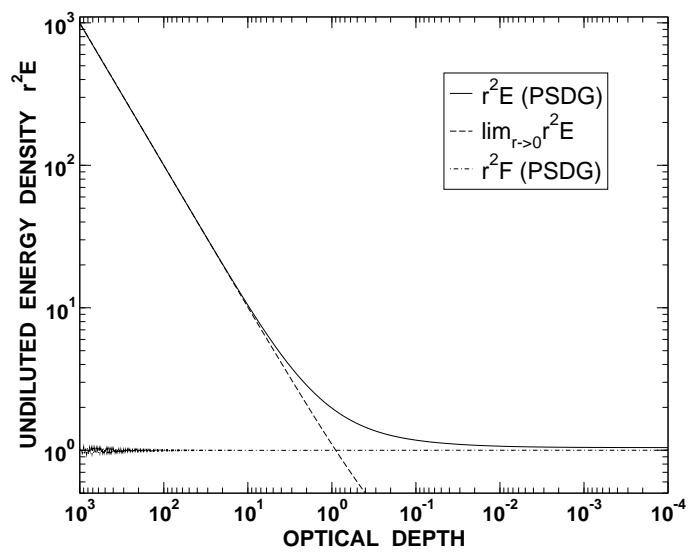

(c)

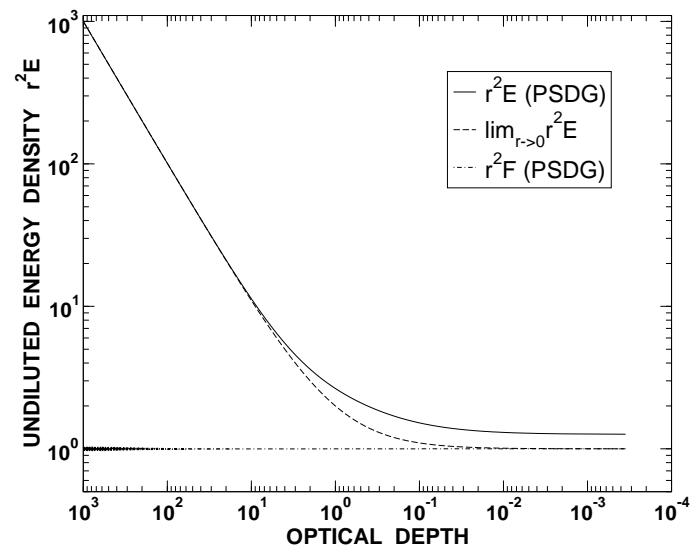

(b)

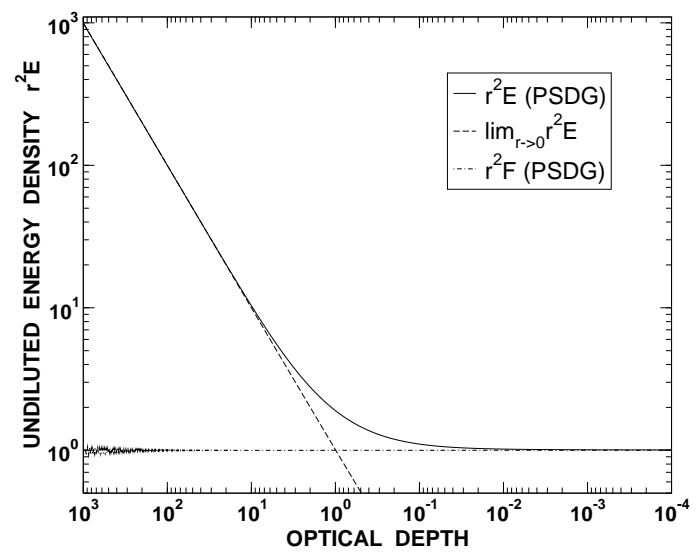

(d)

Fig. 6.9: Undiluted energy density and flux (luminosity) for $n=2$; measured in units of $L_{0} /(4 \pi c)$. Computed luminosity is a constant equal to one. Diffusive behavior for all atmospheres at large optical depths is correctly obtained, while streaming limit at small optical depths for extended atmosphere is correctly approached as the atmosphere grows. (a) $R_{o}=0.1$. (b) $R_{o}=1$. (c) $R_{o}=10$. (d) $R_{o}=100$. 
$\operatorname{det} \boldsymbol{T} \rightarrow 0$ in figure $6.10(\mathrm{~d})$ at $r=R_{o}$. The first invariant is identical to energy density (2.9), thus the other two invariants measure the approach to singularity. Yet another measure of isotropy is the ratio of pressure to energy density, known as the Eddington factor (fig. 6.11). The variation from $1 / 3$, in the diffusive limit, to 1 at the outer boundary of extended atmospheres (sec. 2.1) is often used as a measure of accuracy of calculations. The results obtained here compare well with those (probably less accurate) in the literature (CHAPMAN, 1966, Hummer AND RYBICKI, 1971; RAMPP AND JANKA, 2002; SCHINDER AND BludMAN, 1989).

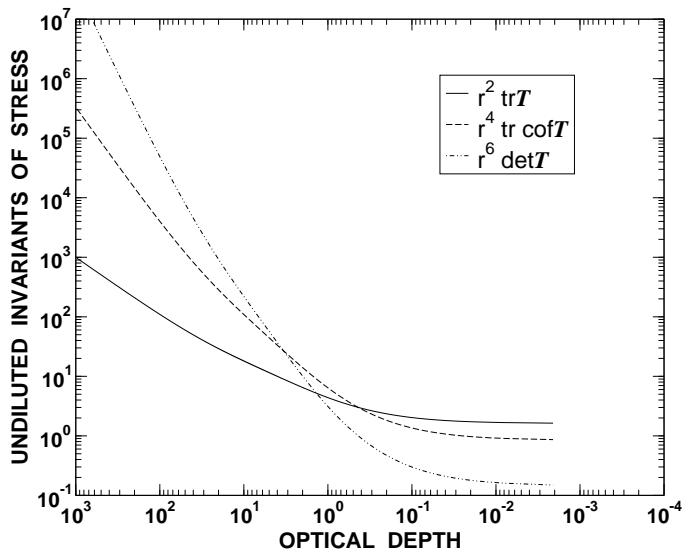

(a)

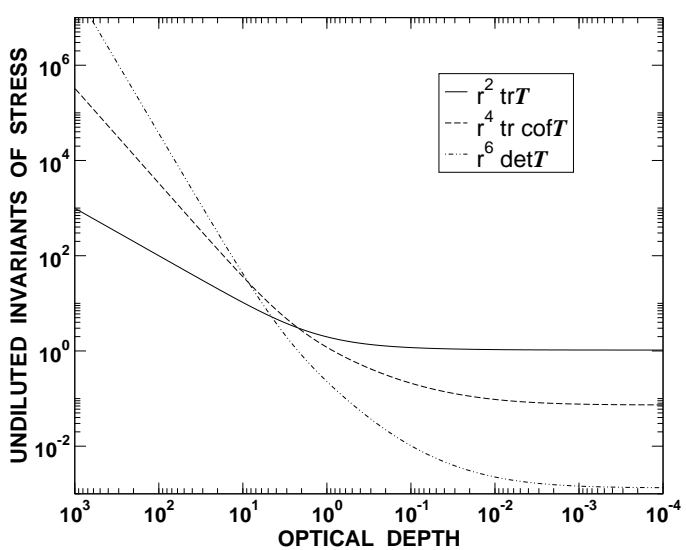

(c)

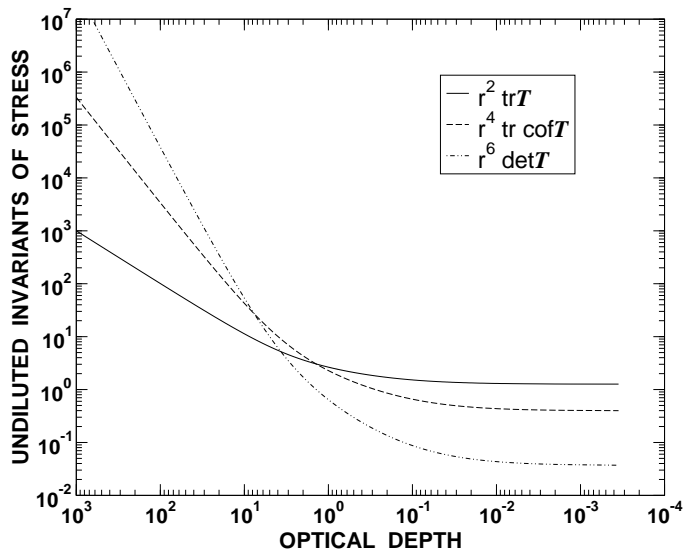

(b)

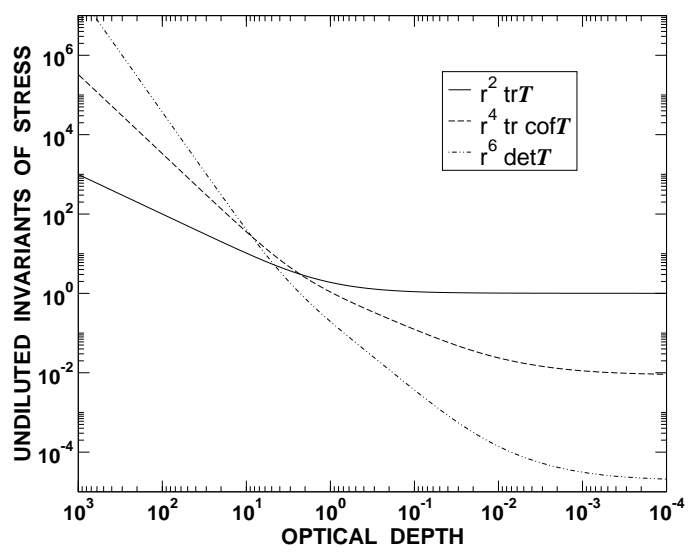

(d)

Fig. 6.10: Undiluted principal invariants of the radiative stress tensor $(n=2)$. (a) $R_{o}=0.1$. (b) $R_{o}=1$. (c) $R_{o}=10$. (d) $R_{o}=100$. Principal invariants measured in units of $L_{0} /(4 \pi c), L_{0} /\left(4 \pi c \alpha^{2 /(n-1)}\right)$, and $L_{0} /\left(4 \pi c \alpha^{4 /(n-1)}\right)$, respectively. 


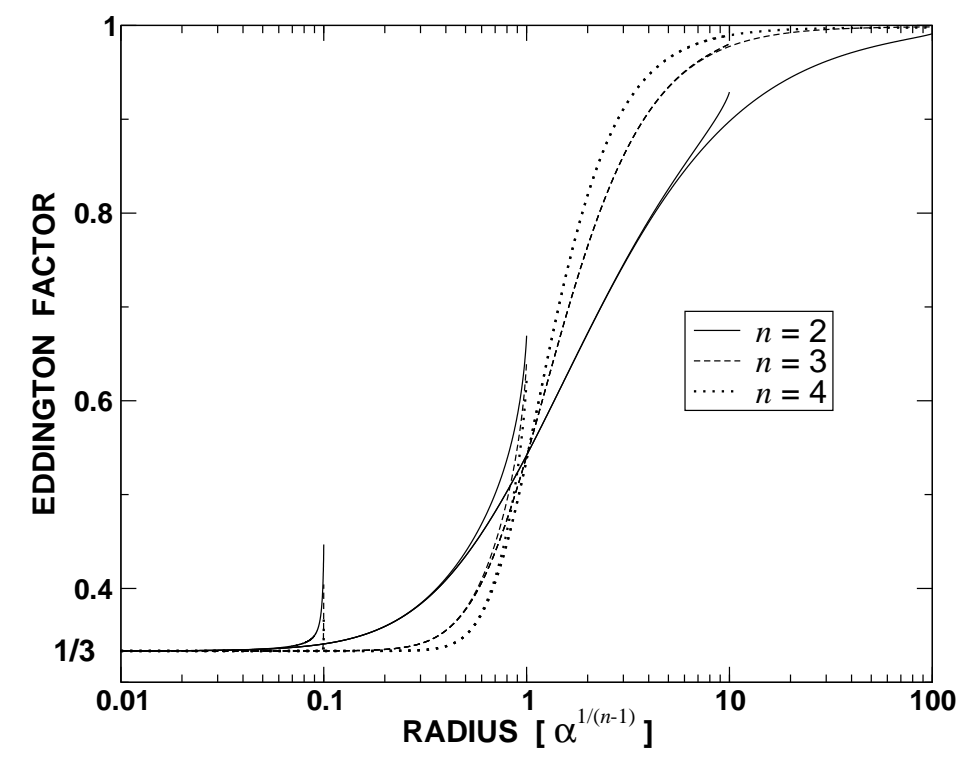

Fig. 6.11: Eddington factors. Ratio of pressure to energy density varying with radius. The end of each curve points to the radius of the outer boundary.

\subsection{Convergence}

Convergence of the iterative scheme (3.1) took place within thirteen iterations for all cases (fig. 6.12). The inner iteration used for refinement resulted in a maximum update norm of about $10^{-14}$. The norm of the first update (sec. 4), used to verify convergence during iterations, dropped monotonically with average convergence order approximately equal to 0.7 (fig. 6.13), implying a reduction of update and residual norms by a factor of five per iteration. The order of convergence is defined as

$$
-\log \frac{\max _{\mathscr{K}_{e} \in \mathscr{T}^{h}}\left\|\delta I_{1}^{(k)} / I^{(k)}\right\|_{\mathrm{H}^{1}\left(\mathscr{K}_{e}\right)}}{\max _{\mathscr{K}_{e} \in \mathscr{T}^{h}}\left\|\delta I_{1}^{(k-1)} / I^{(k-1)}\right\|_{\mathrm{H}^{1}\left(\mathscr{K}_{e}\right)}} .
$$

\section{Conclusions}

An additional source of exploitation of parallelism is available in the computation of the $p$ th moment (4.15). The construction of the moment-neighbors list $\mathscr{N}_{e}^{i}$ (sec. 4.3) can be done independently and in parallel for each Gauss point $\boldsymbol{g}^{i}$ for all finite elements 


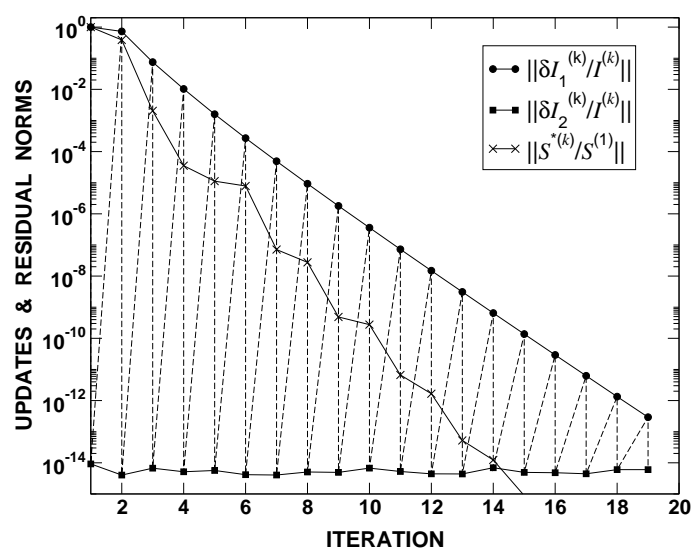

(a)

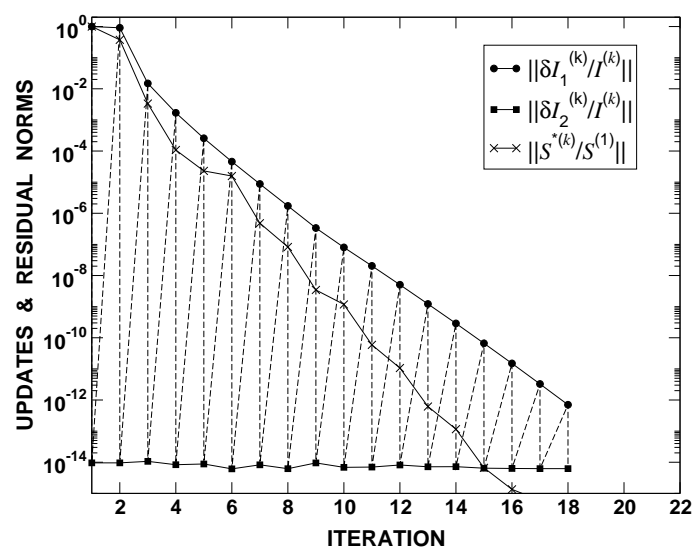

(c)

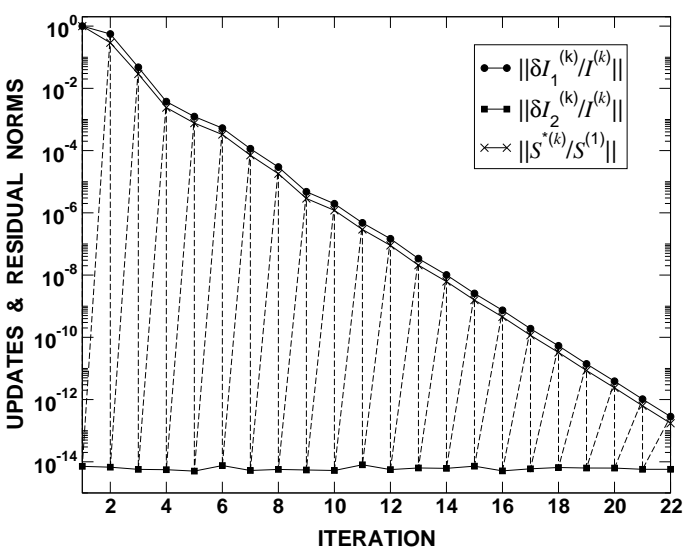

(b)

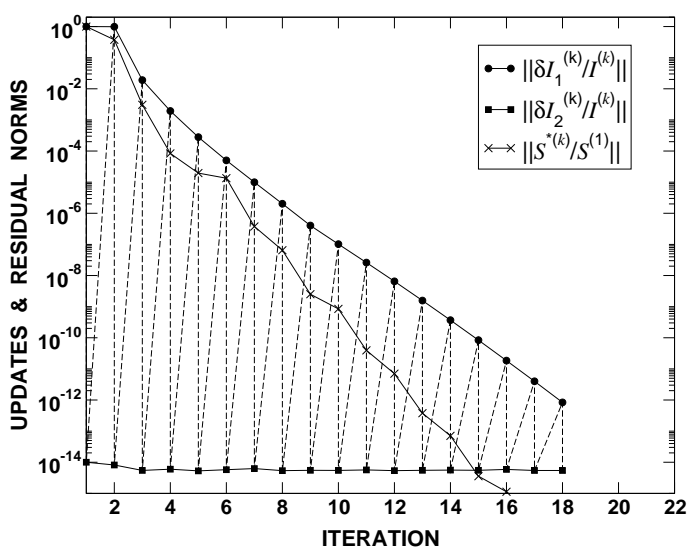

(d)

Fig. 6.12: Updates and Galerkin (weighted) residuals convergence for $n=2$. (a) $R_{o}=0.1$. (b) $R_{o}=1$. (c) $R_{o}=10$. (d) $R_{o}=100$. 


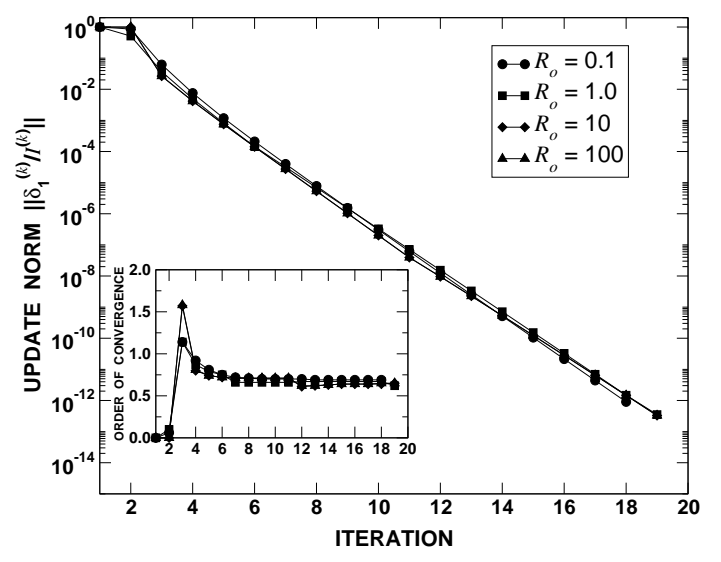

(a)

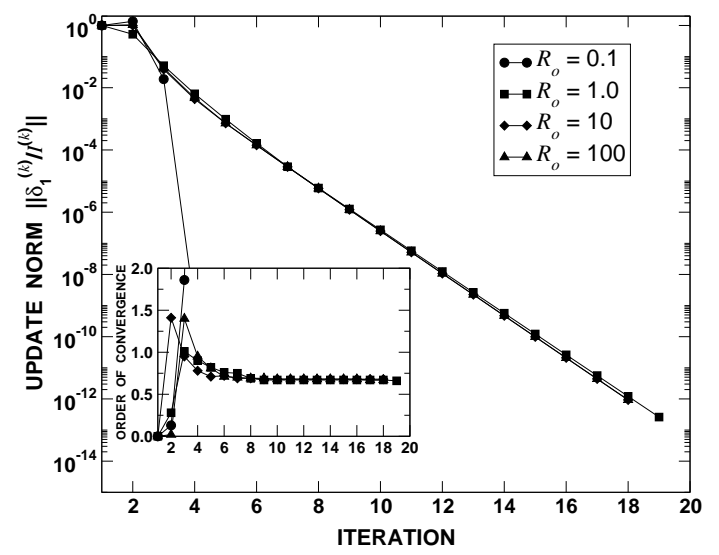

(c)

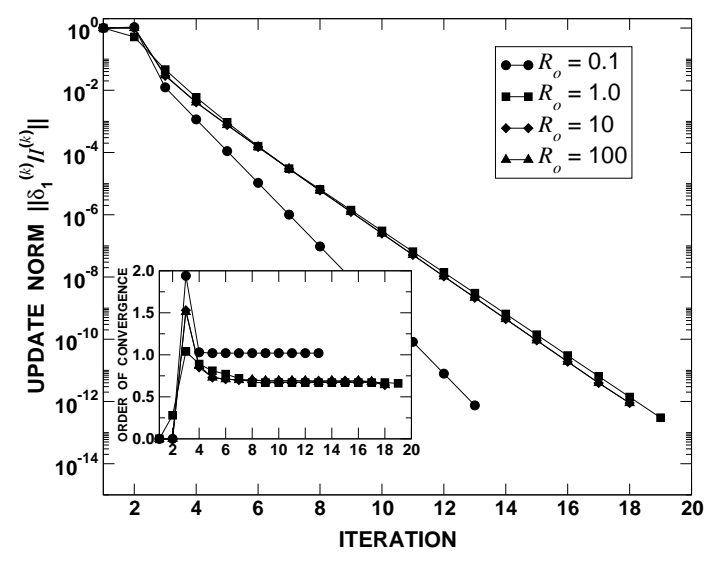

(b)

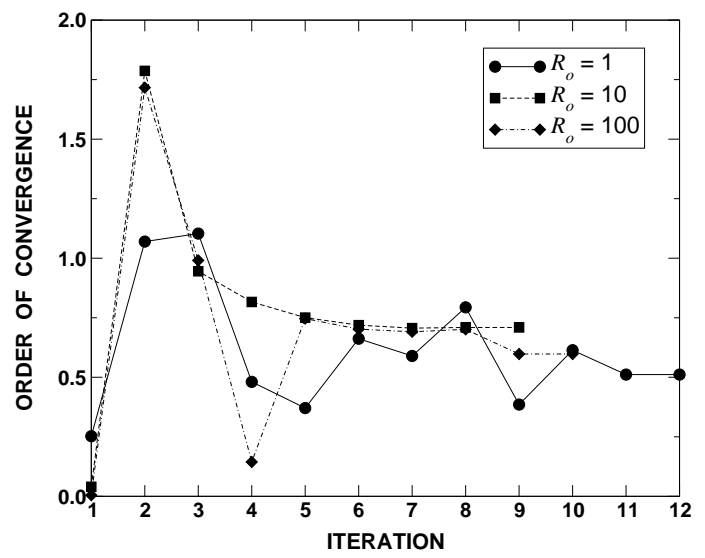

(d)

Fig. 6.13: First update norms and order of convergence 6.1) (inset graphs) for algorithm (3.1) (average order of convergence $\approx 0.7$ ). (a) $n=4$. (b) $n=6$. (c) $n=8$. (d) $n=2$. 
$\mathscr{K}_{e}$. Parallelism will certainly reduce the wall clock time required for computing the moments, but it is unlikely that this step in algorithm 3.1 will ever be faster than the solution of the homogeneous problem (4.1) also solved in parallel. Therefore, it is anticipated that computing the moments of radiation will remain to be a task to pay attention to.

The use of a structured quadrilateral partition of the phase space was instrumental to simplify the solution of (4.10) via computational wave fronts perfectly perpendicular to the radial axis. That was possible because all finite element edges enjoyed the uniflow condition (sec. 4.2). Such condition will not exist for all elements in an unstructured partition, say using triangles. The compelling reason for using unstructured partitions is that the real benefits of the PSDG method will only be realized by taking advantage of the full adaptivity on such elements. Only this feature will set the method apart from any other solution method currently in use for radiative transfer analysis.

On an unstructured partition, some finite elements will have edges with simultaneous inflow and outflow of phase-space radiative flux. The implication is that the solution of (4.10) will not follow from a simple ordering of $\mathscr{T}^{h}$ with successive use of (4.11) for each $\mathscr{T}_{i}^{h} \subset \mathscr{T}^{h}$. Some of the terms in the sum on the right side of (4.10) will need to be moved back to the left side and treated as unknowns. Accordingly, the equation will hold for the set of elements moved to left side as opposed to one single element. The analogous geometric operation entails finding all "clusters" of elements whose combined boundary only allows either inflow or outflow. These clusters will need to be amalgamated into a macro element and treated as a single element. Note that it is always possible to find clusters - the worst case scenario being a cluster with all elements in the finite element partition. It is also noted that clusters will require an implicit solution of the associated algebraic equations which will have an adverse impact on exploitation of computational parallelism. In summary, when using unstructured partitions suitable for adaptivity, a cluster version of (4.10) and (4.11) will be required.

Several interesting investigations on extended atmosphere can follow from this work with the use of a general adaptive PSDG method. In particular, cases incorporating absorption and emission, and polarization (CHANDRASEKHAR, 1960). Another challenging and interesting case is the incorporation of energy spectra which will increase the dimension of the phase space to at least three and eventually to a full six-dimensional phase space. In the above mentioned extensions, the corresponding moments of the extended radiative transfer equation need to be used to correct for any appearing integral term. Thus the iterative scheme (sec. 3) will need to be revisited.

Oak Ridge National Laboratory Technical Report ORNL/TM-2003/072 pp. 1-44 


\title{
Acknowledgments
}

This work was partially supported by the SciDAC (Scientific Discovery through Advanced Computing) program of the Division of Mathematics, Informatics, and Computational Sciences within the Office of Advanced Scientific Computing Research, U.S. Department of Energy under the contract DE-AC05-00OR22725 with UT-Battelle, LLC. Ed d'Azevedo and Tony Mezzacappa involved the author in this research area. Ahmed Khamayseh and Bronson Messer participated on discussions. Computational production-time on IBM and Alpha systems provided by ORNL Center for Computational Sciences.

\author{
OAK Ridge National Laboratory \\ P.O. Box 2008 \\ OAK Ridge, Tennessee, TN 37831-6181 \\ U.S.A. \\ E-mail: dealmeidav@ornl.gov
}

\section{References}

BAL, G. 2000 Spatially varying discrete ordinates methods in xy-geometry. Math. Mod. Meth. Appl. S. 10 1277-303.

Broadwell, J. E. 1964 Shock structure in a simple discrete velocity gas. Phys. Fluids 7 1243-7.

Chandrasekhar, S. 1944 On the radiative equilibrium of a stellar atmosphere. II. Astrophys. J. 100 76-86.

Chandrasekhar, S. 1945 On the radiative equilibrium of a stellar atmosphere. V. Astrophys. J. 101 95-107.

Chandrasekhar, S. 1948 On the radiative equilibrium of a stellar atmosphere. XXIV. Astrophys. J. 108 92-111.

Chandrasekhar, S. 1960 Radiative Transfer. Dover Publications, Inc., New York, New York. Originally published in 1950 by Oxford University Press.

Chapman, R. D. 1966 Radiative transfer in extended stellar atmospheres. Astrophys. J. 143 61-74. 
Chui, E. H. And Raithby, G. D. 1993 Computation of radiant heat transfer on a nonorthogonal mesh using the finite-volume method. Numerical Heat Transfer, Part B 23 269-88.

Ciarlet, P. G. 1978 The Finite Element Method for Elliptic Problems. NorthHolland, Amsterdam.

Cockburn, B., Karniadakis, G. E., and Shu, C.-W. 2000 The development of discontinuous galerkin methods. In B. CockBurn, G. E. Karniadakis, And C.-W. Shu, editors, Discontinuous Galerkin Methods, number 11 in Lecture Notes in Computational Science and Engineering, pages 1-50. Springer, New York.

Hummer, D. G. And Rybicki, G. B. 1971 Radiative transfer in spherically symmetric systems. The conservative grey case. Mon. Not. R. astr. Soc. 152 1-19.

Hummer, D. G. And Rybicki, G. B. 1975 A note on the 'peaking effect' in spherical-geometry transfer problems. Mon. Not. R. astr. Soc. 170 423-7.

Kunasz, P. B. And Hummer, D. G. 1974 Radiative transfer in spherically symmetric systems-IV. Mon. Not. R. astr. Soc. 166 57-78.

Lasaint, P. And Raviart, P. A. 1974 On a finite element method for solving the neutron transport equation. In C. DE Boor, editor, Mathematical Aspects of Finite Elements in Partial Differential Equations, pages 89-123. Academic Press, New York.

Lathrop, K. D. 1968 Ray effects in discrete ordinates equations. Nucl. Sci. Engng $32357-69$.

Lathrop, K. D. 1971 Remedies for ray effects. Nucl. Sci. Engng 45 255-68.

Lewis, E. AND Miller, JR., W. 1984 Computational Methods of Neutron Transport. John Wiley and Sons, New York, New York.

Marchuk, G. I. And Lebedev, V. I. 1986 Numerical methods in the theory of neutron transport. Harwood Academic Publishers, New York, New York, second edition.

Martin, W. R., Yehnert, C. E., Lorence, L., and Duderstadt, J. J. 1981 Phase-space finite element methods applied to the first-order form of the transport equations. Ann. Nucl. Energy 8 633-46.

Mezzacappa, A. 2001 Toward a standard model of core collapse supernovae. Nucl. Phys. A 688 158C-167C. 
Mihalas, D. And Weibel-Minalas, B. 1999 Foundations of Radiation Hydrodynamics. Dover Publications, Inc., New York, New York. Originally published in 1984 by Oxford University Press.

Miller Jr., W. F., Lewis, E. E., And Rossow, E. C. 1973 The application of phase-space finite elements to the two-dimensional neutron transport equation in x-y geometry. Nucl. Sci. Engng 52 12-22.

Mordant, M. 1986 Phase-space finite elements encoded in ZEPHYR for x-y and r-z transport calculations. Prog. Nucl. Energy 18 27-37.

Platkowski, T. And Illner, R. 1988 Discrete velocity models of the boltzmann equation: a survey on the mathematical aspects of the theory. SIAM Review 30 $213-55$.

Raithby, G. D. And Chui, E. H. 1990 A finite-volume method for predicting a radiant heat transfer in enclosures with participating media. ASME Journal of Heat Transfer 112 415-23.

RAMPP, M. AND JANKA, H.-T. 2002 Radiation hydrodynamics with neutrinos: Variable Eddington factor method for core-collapse supernova simulations. $A \& A$ 396 361-92.

Rothman, D. H. And Zaleski, S. 1997 Lattice-Gas Cellular Automata: Simple models of complex hydrodynamics. Cambridge University Press, Cambridge.

Schinder, P. J. And Bludman, S. A. 1989 Radiative transport in spherical static spacetime: General relativistic tangent-ray method for the variable Eddington factors. Astrophys. J. 346 350-65.

Siegel, R. And Howell, J. R. 1992 Thermal Radiation Heat Transfer. Taylor \& Francis, Washington, DC, third edition.

Truesdell, C. 1975 Early kinetic theories of gases. Arch. Hist. Exact Sci. 15 1-66.

Wick, G. C. 1943 Über ebene diffusionsprobleme. Z. Phys. 120 702-18.

Woosley, S. E. And Heger, A. 2002 The evolution and explosion of massive stars. Rev. Mod. Phys. 74 1015-71. 